\title{
TrmB family transcription factor as a thiol-based regulator of oxidative stress response
}

Paula Mondragon $^{1 \#}$, Sungmin Hwang ${ }^{1,2 \#}$, Lakshmi Kasirajan ${ }^{1,5}$, Rebecca Oyetoro ${ }^{1}$, Angelina

Nasthas ${ }^{1}$, Emily Winters ${ }^{1}$, Ricardo L. Couto-Rodriguez ${ }^{1}$, Amy Schmid ${ }^{2,3^{*}}$, and Julie A. Maupin-

Furlow $^{1,4^{*}}$

${ }^{1}$ Department of Microbiology and Cell Science, Institute of Food and Agricultural Sciences,

University of Florida, Gainesville, Florida, USA 32611.

²Department of Biology, Duke University, Durham, NC, 27708, USA.

${ }^{3}$ Center for Genomics and Computational Biology, Duke University, Durham, NC, 27708, USA.

${ }^{4}$ Genetics Institute, University of Florida, Gainesville, Florida, USA 32611.

${ }^{5}$ ICAR-Sugarcane Breeding Institute, Coimbatore, 641007, India

\#These authors contributed equally to this work.

${ }^{*}$ Corresponding authors

Emails: pmondragon@ufl.edu; sungmin.hwang@duke.edu; k.lakshmi@icar.gov.in;

beccatoro@yahoo.com; anasthas@ufl.edu; emily.winters@ufl.edu; r.coutorodriguez@ufl.edu;

amy.schmid@duke.edu; jmaupin@ufl.edu

Running title: TrmB-like thiol-based transcription factor 
Abstract: Oxidative stress causes cellular damage including DNA mutations, protein dysfunction and loss of membrane integrity. Here we discovered TrmB (transcription regulator of mal operon) family proteins (Pfam PF01978) composed of a single winged-helix DNA binding domain (InterPro IPR002831) can function as thiol-based transcriptional regulators of oxidative stress responses. Using the archaeon Haloferax volcanii as a model system, we demonstrate that the TrmB-like OxsR is important for recovery of cells from hypochlorite stress. OxsR is shown to bind specific regions of genomic DNA, particularly during hypochlorite stress. OxsRbound intergenic regions were found proximal to oxidative stress operons including genes associated with thiol relay and low molecular weight thiol biosynthesis. Further analysis of a subset of these sites, revealed OxsR to function during hypochlorite stress as a transcriptional activator and repressor. OxsR was shown to require a conserved cysteine (C24) for function and to use a CG-rich motif upstream of conserved BRE/TATA box promoter elements for transcriptional activation. Protein modeling suggested the C24 is located at a homodimer interface formed by antiparallel $\alpha$ helices, and that oxidation of this cysteine would result in the formation of an intersubunit disulfide bond. This covalent linkage may promote stabilization of an OxsR homodimer with the enhanced DNA binding properties observed in the presence of hypochlorite stress. The phylogenetic distribution TrmB family proteins, like OxsR, that have a single winged-helix DNA binding domain and conserved cysteine residue suggests this type of redox signaling mechanism is widespread in Archaea.

Importance: TrmB-like proteins, while not yet correlated with redox stress, are found in bacteria and widespread in archaea. Here we expand annotation of a large group of TrmB-like single winged-helix DNA binding domain proteins from diverse archaea to function as thiol-based transcriptional regulators of oxidative stress response. Using Haloferax volcanii as a model, we reveal the TrmB-like OxsR functions during hypochlorite stress as a transcriptional activator and repressor of an extensive gene co-expression network associated with thiol relay and other related activities. A conserved cysteine residue of OxxR serves as the thiol-based sensor for this function and likely forms an intersubunit disulfide bond during hypochlorite stress that stabilizes a homodimeric configuration with enhanced DNA binding properties. A CG-rich DNA motif in the promoter region of a subset of sites identified to be OxsR-bound is required for regulation; however, not all sites have this motif suggesting added complexity to the regulatory network. 


\section{Introduction}

Oxidative stress can be exceedingly damaging to cells. Once the levels of reactive species overwhelm the antioxidant capacity of cells, lipid peroxidation, protein denaturation, DNA hydroxylation, and other damaging effects occur that impair cellular viability (1). To survive these challenges, cells must sense and respond to oxidant challenge, shifts in redox balance, and the damage encountered due to oxidative stress. Transcription factors (TFs) that sense and respond to reactive species are found to mediate global changes in gene expression to remedy the damage $(2,3)$. These TFs often have metalloclusters, cofactors, or residues (cysteine, histidine or methionine) that are sensitive to oxidant and serve as "redox switches" $(4,5)$. These switches can turn TF function on or off in coordinating gene co-expression networks associated with electron flow, antioxidant systems and other pathways (6-8).

Archaea have an array of metabolic strategies and physiological adaptations that enable them to sense and respond to extreme environments. TFs are central to sensing and responding to environmental cues and in relaying this signal to coordinate gene-expression networks. Thus, TFs are anticipated to be important in facilitating the ability of archaea to respond and thrive in extreme environments including exposure to reactive species $(9,10)$. TFs with redox switches are diverse and widespread in bacteria and likely to function in archaea to sense oxidant rich conditions. Archaea, while distinct from bacteria in their use a eukaryotic-like basal transcription machinery, have TFs that are often related to bacteria $(9,10)$. This similarity in TFs is due to their evolution which includes shared ancestral proteins and inter-domain horizontal gene transfer events between archaea and bacteria. Thus, it is surprising that the bacterial TFs, such as OhrR (11-13), SarA/MgrA (14), PerR (15), HypR (16), YodB (17), QsrR (18), MosR (19), SarZ (20), OxyR (21-23), SoxR $(24,25)$, and FNR (26), that use redox switches to mediate global alterations of transcriptional networks are not readily identified in archaea. 
The two TFs that have been identified in archaea to use redox switches are from the ArsR family, i.e., MsvR (MTH_1349) and SurR (PF0095). Oxidation of key cysteine residues of these TFs negatively affects their DNA binding activity. SurR responds to elemental sulfur $\left(\mathrm{S}^{0}\right)$, an electron acceptor in the Thermococcales, leading to the deactivation of genes associated with $\mathrm{H}_{2}$ production and the derepression of genes needed for $\mathrm{S}^{0}$ metabolism (27-30). MsvR regulates the transcription of its own promoter and an adjacent operon implicated in the oxidative stress response of methanogens (31-33). MsvR appears exclusive to methanogens, while SurR clusters to the Archaeal Clusters of Orthologous Gene (arCOG) group arCOG01684 (34), suggesting its general function is more widespread.

TrmB (transcription regulator of mal operon) family TFs, though not yet correlated with redox stress, are widespread in archaea and found in bacteria $(9,35)$. TrmB family proteins appear to have undergone an evolutionary expansion in archaea after divergence from bacteria, with homologs accounting for $12 \%$ of the total number of TFs in archaea compared to $0.5 \%$ in bacteria (9). TrmB family TFs have an N-terminal DNA binding domain that is sometimes fused to a C-terminal ligand sensing domain. The ligands that bind to the C-terminal domain can be sugars or other molecular factors. TrmB family TFs with these two domains (the N-terminal DNA binding domain and C-terminal ligand sensing domain) typically function as global transcriptional activators and/or repressors of sugar transport and metabolism including glycolysis, gluconeogenesis, the TCA cycle, amino acid metabolism, methanogenesis, and autotrophic pathways (36-46). TrmB homologs with the single DNA binding domain, while less characterized, appear to function differently than their two-domain counterparts. This variation is exemplified by TrmBL2 (TrmB-like protein 2), an abundant DNA binding protein of the Thermococcales that clusters to arCOG02037. TrmBL2 functions as a global transcriptional repressor and a chromatin binding protein that can rearrange genomic DNA from a conventional histone-bound 'beads-on-a-string' architecture to a thick fibrous structure (34). When compared 
to other TrmB family proteins, TrmBL2 has an expanded function as it can bind single-stranded and double stranded DNA (47).

Identification of TFs and other global regulatory systems used by archaea to sense and respond to reactive species is imperative to provide new insights into hypertolerance mechanisms. Haloarchaea provide a useful resource for this discovery as they inhabit some of the saltiest places on Earth, including hypersaline lakes, marine salterns and brine inclusions, which are high in reactive species $(48,49)$. Compared to most organisms, haloarchaea display an order of magnitude higher tolerance to oxidant rich conditions, which is likely associated with their adaptation to these hypersaline ecosystems $(50,51)$. Haloarchaea employ an unusual 'highsalt-in' strategy in which molar concentrations of potassium and chloride are accumulated intracellularly to maintain osmoprotection and cellular homeostasis (52-55). These high concentrations of chloride promote the generation of reactive chloride species (RCS), reactive oxygen species (ROS), and other stressful agents (56-58). High levels of reactive species are also generated in hypersaline habitats through common cycles of desiccation-rehydration and intense UV radiation $(56,57,59)$. Surprisingly, haloarchaea thrive under these conditions and are often the last remaining communities when the salt concentrations reach saturation (60).

Regulators of oxidative stress responses are identified in haloarchaea; however, the mechanisms of how these regulators sense oxidant remain to be determined. Included among these factors are RosR (VNG0258H) and SHOxi. RosR, a PadR-type TF with a winged helixturn-helix (wHTH) domain, is required for gene expression dynamics during extreme oxidative stress in Halobacterium sp. NRC-1 (61-63). RosR homologs cluster to the arCOG00006 group and are found widespread among haloarchaea suggesting a common mechanism, yet the residues that may directly sense oxidant are not readily identifiable. SHOxi is a small noncoding RNA in Haloferax volcanii that impacts redox balance by destabilizing malic enzyme 
mRNA and, thus, decreasing the ratio of $\mathrm{NAD}^{+} / \mathrm{NADH}(64)$. SHOxi is upregulated during oxidative stress, suggesting other factors serve upstream of this response to sense the redox status of cells.

Here we discover that TrmB family proteins can function as TFs that sense redox status and facilitate the recovery of cells from hypochlorite stress. Using Haloferax volcanii as a model system, we targeted the TrmB family protein HVO_2970 of arCOG02242 for study, as it undergoes a several-fold increase in protein abundance after exposure of cells to hypochlorite stress as determined by stable isotope labelling of amino acids in cell culture (SILAC)-based proteomic analysis (65). Here we reveal HVO_2970 is a thiol-based TF required for recovery of cells from hypochlorite stress and, thus, it is named OxsR, for oxidative stress responsive regulator. By coupling chromatin immunoprecipitation sequencing (ChIP-seq) and quantitative real-time polymerase chain reaction (qRT-PCR) analyses, we demonstrate that OxsR regulates the expression of genes associated with thiol relay, low molecular weight thiol synthesis, and other related functions during hypochlorite stress. OxsR functions as a transcriptional activator and repressor, with these distinctions found to correlate with the presence and positioning of a CG-rich motif relative to the BRE/TATA box promoter consensus sequence. By site-directed mutagenesis, a cysteine residue $(\mathrm{C} 24)$ oriented at a predicted homodimer interface was demonstrated to be important for OxsR function. This cysteine residue was conserved among TrmB-like single DNA binding domain proteins from diverse archaeal phyla suggesting this type of redox signaling mechanism is widespread and represents a new type of thiol-based TF. 


\section{Results}

OxsR phylogenetic distribution. OxsR is member of the TrmB family (Pfam PF01978). Like most archaea, $H$. volcanii encodes multiple TrmB family proteins with all thirteen harboring an N-terminal DNA binding domain (IPR002831) classified to the winged helix-turn-helix (wHTH) superfamily (IPR036388) (Figure 1A). However, only five of these proteins were fused to a Cterminal ligand sensing domain (IPR021586). The remaining eight proteins had a single DNA binding domain including the smallest member of this group: OxsR (123 aa, $14 \mathrm{kDa})$. Such single-domain TrmB family proteins are widespread among archaeal phyla yet poorly understood in function, while two-domain TrmB homologs with a C-terminal ligand binding domain are more commonly studied but not as widely distributed (Figure 1B). Within the TrmB family, OxsR is classified to the archaeal cluster of orthologous genes arCOG02242 (66) and by BlastP comparison is related to homologs of the Euryarchaeota, Crenarchaeota and Asgard archaea (Figure 1B). OxsR does not share close relationship to bacterial members of the TrmB family, which are less common than those of archaea (9). Overall, OxsR is a TrmB family protein that has a single wHTH domain and appears conserved in diverse archaeal phyla.

OxsR is important for recovery of $\boldsymbol{H}$. volcanii from hypochlorite stress. To examine the biological role of OxsR, the following strains were constructed. The oxsR gene was deleted from the $H$. volcanii genome (H26 parent) by markerless deletion (Figure S1, Table S1). The resulting $\Delta o x s R$ mutant was subsequently transformed with plasmids carrying the oxs $R$ gene and the empty vector control. In addition, a strain for OxsR pull-down assays was generated by integration of the hemagglutinin (HA) coding sequence at the 3 ' end of oxs $R$ on the genome. The oxsR-HA integrant strain was also modified to include a C24A mutation discussed in a later section. The strains constructed in this manner were subsequently examined for recovery from hypochlorite stress. All strains were found to be of comparable growth rate (1.7-1.9 h generation time) when cultured under standard conditions in glycerol minimal medium (GMM) (Figure 2A). 
By contrast, differences in strain recovery were observed after hypochlorite treatment (Figure 2B). The parent and HA-tagged strains were found to fully recover from hypochlorite stress after an $88 \pm 8 \mathrm{~h}$ lag (Figure 2B). By contrast, the $\triangle o x s R$ mutant displayed no growth (curve 1, 8 replicates) or recovered after an extended lag (117 \pm 23 h) (curve 2, 4 replicates) under these same conditions (Figure 2B). All replicates of the mutant retained the markerless deletion of the oxs $R$ gene, consistent with whole genome resequencing analysis which revealed oxs $R$ to be deleted from all genomic copies (Figure S2A-C, Table S1). One distinction was identified among the $\triangle$ oxs $R$ mutant replicates: those that that did not recover from hypochlorite stress initially (curve 1) were no longer viable, whereas the replicates that displayed an extended lag (curve 2) were viable, but no longer able to recover after exposure to a second dose of hypochlorite and were not viable once this stress agent was removed (Figure S2D). When performing complementation assays, the $\Delta o x s R$ mutant was found to be uniformly hypersensitive to hypochlorite in the presence of the empty vector, suggesting that the plasmid posed an extra burden to the cells under these conditions (Figure 2B). Expression of the oxs $R$ gene from this multicopy plasmid restored the $\Delta$ oxs $R$ mutant to recover from the hypochlorite stress at parental levels (Figure 2B). This finding suggests that the second site point mutation observed in the $\Delta$ oxs $R$ genome sequence (non-coding intergenic $\mathrm{G}>\mathrm{A}$ mutation between hvo_RS01570 and hvo_RS01575, Table S1) was not the source of the observed hypochlorite recovery defect of $\Delta$ oxsR. Based on these results, OxsR is important for the recovery of $H$. volcanii from hypochlorite stress. Furthermore, the minimal effect of the C-terminal HA-tag on OxsR function during hypochlorite stress (Figure 2B) indicated this construct could be used for downstream immunoprecipitation analysis.

OxsR binds specific regions on the $\boldsymbol{H}$. volcanii genome. OxsR was investigated for its ability to bind specific and/or nonspecific regions of $H$. volcanii genomic DNA by combining chromatin immunoprecipitation with massively parallel DNA sequencing (ChIP-seq). The parent (H26) and 
oxsR-HA tagged strains were grown to log-phase in GMM and then treated with hypochlorite or a mock control prior to the ChIP-seq analysis (see Methods for details). By this approach, OxsR-bound sites were investigated over the chromosome and endogenous plasmids. A total of 29 and 130 sites were found to be putative OxsR interacting regions in the absence and presence of oxidative stress, respectively (Figure S3, Table S2). Although many peaks (63\%) were located within the coding sequences of genes, OxsR was also found to bind distinct intergenic regions of the genome. Most of the OxsR-bound intergenic sites (49 of 59 total) were detected only in the hypochlorite-treated cells with six operons having two 5 ' binding sites. Of the remaining intergenic sites, seven were identified irrespective of the treatment and three were detected only in the absence of hypochlorite (Table S2). When analyzing the genes adjacent to the OxsR-bound regions by arCOG gene functional analysis (67), nearly half (44\%, hypergeometric test $p$-value of enrichment relative to genomic background $<1.1 \times 10^{-2}$ ) of the encoded proteins clustered to the arCOG [S] group of unknown function, thus, providing limited insight (Figure 3). However, $12 \%$ of the proteins clustered to the arCOG [O] group associated with post-translational modification, protein turnover, and chaperone functions, including gene homologs associated with thiol relay (e.g., thioredoxin, thioredoxin-like and disulfide oxidoreductase activity, Table S2); and 3\% to [I] (lipid transport and metabolism, $p<6.1 \times 10^{-5}$ ). Additional functional analysis of the ChIP-seq associated genes by STRING (11.5)(68) corroborated arCOG findings (false discovery rates $<0.0374$; Figure S4). Further inspection of the sites strongly enriched for OxsR binding in intergenic regions ("ChIP-seq high peaks" with height $>1000$-fold enrichment relative to the input control, 27 of 59 total, Table S2) revealed that at least seven of the linked genes were associated with the synthesis of low molecular weight thiols and thiol relay systems (Table 1). HVO_1043, a member of the DUF1684 family, was included in this thiol relay group, as 3D-modeling and multiple amino acid sequence alignment revealed a conserved $\mathrm{Cx}_{7} \mathrm{C}$ motif with the thiol groups of these cysteines in a proximity typical of function in thiol relay (Figure S5). Overall, these results reveal OxsR is a TF 
homolog that binds specific regions of genomic DNA during redox stress. Of the intergenic regions bound by OxsR, an enrichment in the promoter regions of genes associated with protein quality control, thiol relay and other related functions predicted to be important in the recovery from hypochlorite stress was observed.

OxsR functions as an apparent transcription factor. Five genes were selected from the ChIP-seq high peak group to determine whether OxsR has an impact on their transcript abundance. In addition, oxsR (hvo_2970) was included to examine how its transcript levels may correlate with the 3-fold increase in OxsR protein abundance previously identified during hypochlorite stress (69). Transcript levels were monitored by real-time quantitative reverse transcription polymerase chain reaction (RT-qPCR) in the parent and $\Delta$ oxs $R$ mutant after treatment with hypochlorite or a mock control $(t=0)$ (Figure 4). Four of the five genes examined from the ChIP-seq dataset were found to be upregulated in the parent after exposure to hypochlorite including the transcripts of: i) hvo_0811 and hvo_1043, which displayed a rapid increase; and ii) hvo_0040 and hvo_0337, which were more delayed in their upregulation. The exception was hvo_0039, whose expression remained relatively constant in the parent strain regardless of time or treatment. Hypochlorite stress was also found to stimulate a transient increase in the oxs $R$ transcript levels which may in part account for the 3-fold increase in OxsR protein abundance. When comparing the transcript profiles, the $\Delta o x s R$ mutation was found to impact the transcript abundance of all genes examined. Instead of the increased transcript abundance observed in the parent after treatment, when examining the $\Delta$ oxs $R$ mutant: the hvo_0040 and hvo_1043 transcripts were detected at a constitutively low level throughout the time course, while the hvo_0811 and hvo_0037transcripts were only modestly increased in abundance in the early stages of treatment. Counter to the other genes, the hvo_0039 transcripts were found to be of greater abundance in the early stages of hypochlorite treatment in the $\Delta$ oxsR mutant compared to the parent. Overall, the qRT-PCR results suggest that after 
cells are exposed to hypochlorite, OxsR can act as a transcriptional activator (e.g., hvo_0040, hvo_0337, hvo_0811 and hvo_1043 regulation) as well as a repressor (e.g., hvo_0039 regulation). Furthermore, the 3-fold increase in OxsR protein abundance during hypochlorite stress may in part be due to an increase in transcript level.

\section{Conserved DNA motif in the 5' region of a subset of genes identified in the OxsR ChIP.}

seq dataset. De novo motif analysis of the DNA sequences of the intergenic regions identified by ChIP-seq was performed. This analysis was executed to identify conserved DNA motifs in the OxsR-bound intergenic sites and to determine whether the location of these motifs relative to the core promoters correlated with expression change. Similar regions from related haloarchaeal genomes were included in the analysis to enhance DNA motif identification. DNA motifs predicted by MEME MAST (70) to be common to the datasets were subsequently used to scan the $H$. volcanii genome by FIMO (71). FIMO analysis was performed: i) to identify the location of the DNA motifs on the $H$. volcanii genome; and ii) to calculate the significance of the findings in terms of false discovery rate ( $q$-value) and probability ( $p$-value). The FIMO identified sites were then compared to the current ChIP-seq and previously published SILAC datasets, with the latter based on the intergenic regions 5' of genes encoding proteins of differential abundance after hypochlorite treatment as detected by quantitative SILAC-based MS analysis (69). A semi-palindromic DNA motif with evenly spaced CG repeats, $\underline{\text { CGGnCGnGCG, was }}$ identified within OxsR-bound regions (where $\mathrm{n}$ and underline represent the bases of low conservation and palindrome, respectively, E-value $2.5 \times 10^{-132}$; Figure 5A). This motif was detected at 89 sites on the $H$. volcanii genome at a $p$-value $<0.00001$; with about one-third of the sites corresponding to 5' regions associated with the ChIP-seq and SILAC datasets. Of the top seven sites identified at a $q$-value $<0.05$, six clustered to at least one of the two datasets (Figure 5B). Further analysis of the top sites revealed the palindromic DNA motif of CG-repeats was generally positioned 5' of BRE/TATA box consensus sequences presumed to serve as 
RNA polymerase binding sites; as depicted for hvo_1043, hvo_1342, hvo_1668, and hvo_0040 regions (Figure S6A-D). The exception was hvo_0039, which immediately 5' of its start codon had a CG-rich region that was not closely related in sequence to the CG repeat but was conserved in diverse Haloferax species (Figure S6D, upper), which may explain the repressive function of OxsR on the transcript levels of this gene. Thus, the positioning of the CG-rich motifs in relation to the basal promoter element was consistent with the up- or down-regulation in transcript levels observed for these genes by qRT-PCR (previous section). However, the CGrich motif did not fully explain all OxsR-dependent activities as: 1) not all intergenic regions bound by OxsR have this CG-repeat motif and 2) the 12-fold increase observed for hvo_0337 (glutaredoxin) transcripts during hypochlorite stress requires oxs $R$, yet the gene does not encode the CG-rich motif in its promoter region. Nonetheless, these results suggest for a subset of genes that placement of a CG-rich repeat upstream (e.g., hvo_0811, hvo_0040 and hvo_1043) or downstream (e.g., hvo_0039) of the BRE/TATA box promoter consensus element may facilitate the ability of OxsR activate or repress gene expression in the presence of hypochlorite, respectively.

\section{CG-rich motif required for the OxsR-dependent increase in hvo_1043 transcript levels}

after exposure to $\mathrm{HOCl}$. To determine if the CG-rich DNA motif was important for the observed OxsR-dependent increase in hvo_1043 transcript abundance during hypochlorite stress, a mutagenesis approach was used. Strains with deletions in the CG-rich motif upstream of the BRE/TATA box consensus sequence of the hvo_1043 operon were constructed and compared to the H26 parent and $\Delta$ oxsR mutant by qRT-PCR (Figure 5C). A $\Delta h v o \_1043$ coding sequence mutant was also constructed as a negative control. By this approach, a 40-fold increase in the abundance of hvo_1043 transcripts was observed after 15 min of hypochlorite treatment in the H26 parent that was not apparent in the $\triangle 0 x s R$ or the CG-rich motif mutant strains (Figure 5C). The hvo_1043 transcripts were detected only at basal levels in the $\Delta$ oxsR and CG-motif mutant 
strains. As expected, hvo_1043 transcripts were undetectable in the $\Delta h v o \_1043$ mutant. As an added control, the transcripts of other genes (hvo_0040 and hvo_0337) were confirmed to be unaffected by the CG-rich motif deletions that were specifically integrated upstream of hvo_1043. Based on these results, the CG-rich motif identified upstream of the BRE/TATA box consensus sequence of hvo_1043 appears important for transcriptional activation of this operon by OxsR during hypochlorite stress. Because of the close sequence similarity of this GC-rich motif upstream of other OxsR-bound operons, we hypothesize that OxsR may bind this motif to regulate expression of at least a subset of genes within its regulon.

Conserved residues and 3D-structural modeling of OxsR. As OxsR was associated with recovery from hypochlorite, which is a potent oxidant, the primary amino acid sequence of OxsR was inspected for conserved residues that may sense oxidant, such as Cys, His and Met (4). Multiple amino acid sequence alignment revealed that a cysteine (corresponding to OxsR C24) was highly conserved in the $\mathrm{N}$-terminal region of OxsR homologs from diverse archaea (Figure 6A). OxsR was further analyzed by 3D-modeling to predict the location of C24 in the protein structure. The 3D-structure of OxsR residues 18-121 (104 of 123 aa total; 85\%) was modeled at $>99.8 \%$ confidence using the Phyre2 web portal (72). OxsR residues 1-123 were also modeled by RoseTTAfold (73). The alignment scores of the two OxsR 3D-models were at a mean square deviation (RMSD) of $3.47 \AA$ and template modelling (TM)-score of 0.52 , suggesting the models were generally related. The major exceptions were: i) residues $26-46$ which were structurally undefined in the Phyre2-model and ii) residues 1-17 which were not modeled by Phyre2 and found in multiple configurations by RoseTTAfold. The highest scoring template by Phyre2 was the X-ray crystal structure of Methanosarcina mazei MM_1094 (PDB: 3R0A), an uncharacterized TrmB family member of arCOG02242, which has two intersubunit disulfide bonds formed between $\mathrm{C} 6$ and $\mathrm{C} 17$. The single cysteine (C24) of OxsR aligned with $\mathrm{C} 17$ of MM_1094. The OxsR quaternary structure was also predicted by comparison to two other high 
scoring (>99\% confidence) templates including: an assembly of X-ray crystal structures of the Sulfolobus acdiocaldarius AbfR2 (Saci_1223) (PDB: 6CMV)(74) and Streptococcus pneumoniae FabT in complex with DNA (PDB: 6JBX)(75). The quaternary model generated by this approach suggested OxsR could form a homodimer linked by an intersubunit disulfide bridge at $\mathrm{C} 24$ that would join anti-parallel $\alpha$ helices of the two subunits (Figure 6B). A separate homodimer interface formed between two anti-parallel $\alpha$ helices at the C-terminus of OxsR was also predicted.

Cysteine residue is important for OxsR function. To determine whether OxsR C24 is important for sensing oxidative stress, the oxs $R$ genomic locus was modified by site-directed mutagenesis. A strain was constructed to express OxsR C24A with a C-terminal HA tag (OxsRHA C24A) from the oxsR locus similarly to the OxsR-HA form. Using this strain, the role of the conserved C24 was monitored based on: i) the recovery of cells from hypochlorite stress and ii) the relative fold-change in hvo_1043 transcript abundance after exposure of these cells to hypochlorite. The single amino acid exchange of OxsR from C24A reduced the ability of cells to recover from hypochlorite stress, while having little if any impact on growth in the presence of the mock control (Figure 2AB). When using hvo_1043 transcripts to monitor OxsR C24 function, the oxsR-HA C24A integrant strain was found to be severely impaired in its ability to upregulate the abundance of hvo_1043 transcripts in the presence of hypochlorite, to a level comparable to the $\Delta$ oxsR mutant (Figure 7B). By contrast, the parent $(\mathrm{H} 26)$ and the OxsR-HA integrant strains displayed a robust increase in hvo_1043 transcript levels when exposed to hypochlorite (Figure 7B). To determine whether the C24A had an impact on OxsR protein abundance in the cell, the levels of OxsR-HA and OxsR-HA C24A were compared by anti-HA tag immunoblotting analysis (Figure 7C). The anti-HA antibodies were found to be specific to the strains expressing OxsR-HA variants, as no signal was detected in the parent strain devoid of the HA tag. Furthermore, on visual inspection of the immunoblots, the OxsR-HA C24A and 
OxsR-HA were comparable in protein abundance, suggesting the C24A modification did not impact OxsR expression or stability. While amino acid exchange at $\mathrm{C} 24$ did not alter OxsR protein abundance, it did eliminate the ability of OxsR to facilitate the recovery of cells and upregulate the level of hvo_1043 transcript during hypochlorite stress. Thus, C24 appears to be important for OxsR function as a transcriptional activator when strong oxidants of cellular thiols are introduced into the environment.

\section{Discussion}

Here we advance knowledge of oxidative stress signaling in prokaryotes by associating TrmBlike single winged-helix DNA binding domain proteins from diverse archaea as thiol-based transcriptional regulators of oxidative stress response. TrmB is a large and diverse protein family that accounts for over $10 \%$ of the total TFs in archaea and $0.5 \%$ of the TFs in bacteria; however, thiol-based sensory mechanisms were not previously reported for this family. Using the TrmB-like OxsR of Haloferax volcanii as a model, we demonstrate that this protein functions as a transcriptional activator and repressor of a large gene co-expression network associated with oxidative stress response. A conserved cysteine residue serves as the thiol-based sensor for this function and likely forms an intersubunit disulfide bond during hypochlorite stress that stabilizes a homodimeric configuration of OxsR with enhanced DNA binding properties.

Our study relies upon $H$. volcanii cells grown in glycerol minimal medium and exposed to hypochlorite. Thus, the antioxidants present in complex, undefined medium did not complicate our experiments. The hypochlorite used for the environmental cue is a reactive species common to biological systems of high chloride concentration (57). Furthermore, the carbon and energy source, glycerol, was of relevance to hypersaline habitats, where algae accumulate large amounts of glycerol for osmotic stabilization, which is released into the environment (76) for use by heterotrophs such as $H$. volcanii which prefers glycerol over glucose (77). 
Evidence suggests OxsR binds a conserved CG-rich DNA motif in promoters of select genes. Promoters with CG-rich motifs located 5' of the TATA box and BRE consensus were activated by OxsR, whereas those 3' were repressed. These data suggest that the motif may serve as an OxsR binding site and that the positioning of this site in relation to core promoters (TATA binding protein / transcription factor B binding sites) may determine whether OxsR functions as an activator or repressor $(78,79)$.

While the CG-rich DNA motif is common to many of the sites bound by OxsR, not all of the OxsR-bound sites share this motif. One possible explanation for this finding is that other protein factors could associate with OxsR and influence the type of binding site recognized by this TF. Consistent with this hypothesis, some gene induction at early time points following exposure to hypochlorite is still observed in the $\Delta$ oxsR strain for certain OxsR activated genes (hvo_0811), and late repression is possible for other genes (hvo_0039), invoking the involvement of another regulator. One candidate is HVO_1360, a small TrmB family protein that shares $28 \%$ amino acid identity with OxsR and similarly clusters to arCOG02242 (Figure S7A). 3D-structural modeling suggests HVO_1360 forms a wHTH domain flanked on each side by two $\alpha$-helices much like OxsR (Figure S7). Thus, HVO_1360 could potentially form a heterodimer with OxsR that is stabilized by an intersubunit disulfide bond at the anti-parallel $\alpha$ interface of these two subunits (OxsR C24 bound to C21 or C15 of HVO_1360). Formation of this type of complex could alter the DNA sites bound by OxsR and present in the ChIP-seq dataset. An alternative explanation is that OxsR has an extensive DNA footprint with limited to no recognizable motif as seen for other TFs (discussed in detail below).

TFs with thiol-based redox switches are common in bacteria $(4,5)$. These TFs are generally classified into 1-Cys and 2-Cys based on sensing through one or two cysteine residues, 
respectively. Methionine or histidine residues, flavin cofactors, iron, iron-sulfur clusters, and heme centers are also used in bacterial TFs to sense redox status and can be found as added sensors in the thiol-based TFs. ROS or other redox-active compounds can cause specific modifications that lead to conformational changes of these TFs and result in the loss, gain or alteration of their DNA binding activity. Examples of bacterial TF thiol-based modifications include inter- or intra-subunit disulfide bond formation, S-thiolation (mixed disulfides of proteins and low molecular weight thiols), cysteine phosphorylation, and thiol-S-alkylation. These modifications can lead to transcriptional activation, repression or derepression depending on the TF. Many bacterial TFs with thiol-based switches are inactivated by redox stress leading to transcriptional derepression or deactivation, e.g., 1-Cys $\operatorname{OhrR}(11,12)$, 2-Cys OhrR (13), SarA/MgrA (14), PerR (15), HypR (16), YodB (17), QsrR (18), MosR (19), SarZ (20). One of the most versatile groups of thiol-based bacterial TFs activated by stress are the OxyR homologs of the LysR family including 2-Cys and 1-Cys type. In the presence of peroxide, nitric oxide ('NO) or oxidized glutathione (GSSG), OxyR forms intramolecular disulfide bonds (2-Cys OxyR) or other post-translational thiol modifications that can transform the TF into a transcriptional activator (21) or repressor (22) or lead to derepression (23). In the oxidized state, OxyR forms tetramers that bind DNA with an extensive footprint ( $50 \mathrm{bp})$ composed of repeating spaced elements of limited sequence similarity $(80,81)$. The well-known bacterial SoxRS operon is also activated by oxidative stress, with its DNA binding activity triggered by the oxidation or nitrosylation of [2Fe-2S] clusters $(24,25)$. By contrast, bacterial FNR homologs require the presence of an intact 4Fe-4S cluster (otherwise disrupted by oxygen) for function as global transcription regulator when oxygen becomes scarce (26).

Thiol-based redox switch TFs are also found in archaea and eukaryotes. Prior to our work, thiolbased redox switches were identified in TFs of archaea but limited to the ArsR family of transcriptional repressors MsvR (31-33) and SurR (27-30). Upon shifts to oxidizing conditions, 
these 2- and 5-Cys TFs form intra- and intersubunit disulfide bonds (SurR (29) and MsvR (32), respectively) that result in TF inactivation and transcriptional deactivation and/or derepression. In yeast, Yap1 is identified as a basic leucine zipper (bZIP) TF that uses a thiol-based redox switch to function as a central regulator of oxidative stress response pathways $(82,83)$. In the presence of $\mathrm{H}_{2} \mathrm{O}_{2}$, Yap1 forms intramolecular disulfide bonds that alter its conformation and mask its nuclear export signal. These changes promote the accumulation of Yap1 in the nucleus and stimulate transcriptional activation of its regulon (84), with formation of an intermolecular thiol intermediate between Yap1 and the thiol peroxidase Gpx3 inhibiting this activity (85). Thus, of the various domains of life, thiol-based TFs that are activated by oxidative stress and mediate a global response have yet to be reported in archaea.

Small wHTH domain proteins that cluster to arCOG02242 similarly to OxsR are characterized in Crenarchaeota including Lsr14, Smj12 and others (Table S3). Of these proteins, Lsr14 purifies as a homodimer and forms large footprints (> $50 \mathrm{bp}$ ) over its own promoter and the alcohol dehydrogenase (adh) promoter suggesting it functions as a transcriptional repressor (86-88). The size of the footprints suggests that Lsr14 assembles from a homodimer into higher-order complexes in the promoter region (88). Lsr14 is also found to associate with other DNA binding proteins, such as the benzaldehyde-activated TF (Bald) and the chromatin remodeling proteins Sso7d and Sso10b (Alba), suggesting additional levels of regulation (88). While of low protein abundance in the cell, the related Smj12 displays activities which suggest it has a role in chromatin remodeling including binding DNA non-specifically, stabilizing the DNA double helix, and introducing positive supercoiling in DNA (89). More recently, Lrs14, AbfR1 and AbfR2 proteins of this arCOG group are correlated with biofilm formation, adhesion and motility (90, 91), with phosphorylation of AbfR1 Y84 and S87 found important for its binding to promoter regions (92). 
Based on this study, we find OxsR to have common and distinct properties with the arCOG02242 group representatives that have been characterized. While a CG-rich motif appears important for the DNA binding and transcriptional activity of OxsR at promoter regions, the ChIP-seq enrichment peaks for OxsR were on average $700 \mathrm{bp}$ (Table S2), which is wider than the peaks observed for other previously characterized halophilic TFs $(40,93)$. Furthermore, our ChIP-seq analysis revealed six operons to have multiple 5 ' intergenic sites bound to OxsR (i.e., hvo_A0618, hvo_2758, hvo_1875, hvo_0198, hvo_1043 and hvo_1342). These results suggest OxsR homodimers may form higher order structures and bind DNA with large footprints similarly to Lsr14. OxsR does not appear to be a highly abundant protein that non-specifically binds DNA, as specific sites were identified by ChIP-seq analysis, and OxsR-HA could not be detected by western blotting without prior enrichment by immunoprecipitation, which contrasts with the HA-tagged TF GlpR (94). Furthermore, unlike abundant proteins such as proteasomes, OxsR is detected in only four of the six whole proteome datasets reported in the Archaeal Proteome Project (ArcPP) (95). The most important distinction of OxsR with the previously characterized arCOG02242 members is that it is found to use a thiol-based sensor in the transcriptional response associated with recovery from hypochlorite stress.

Post-translational modification (PTM) appears important in regulating the activity of the arCOG02242 TFs. We find most members of this arCOG group have conserved cysteine residues located at predicted homodimer interfaces formed by either the antiparallel $\alpha 1$ and/or a5 helices (Figure 6, Table S3). These cysteine residues form intersubunit and intrasubunit disulfide bonds in the X-ray crystal structures of Sto12a and MM_1094 and, thus, could generally serve as redox sensors that influence TF homodimer stability. Ser/Thr and Tyr phosphorylation provides an additional type of post-translational regulation to consider in the signaling pathway of TFs of the TrmB family arCOG02242 group (92). While the Y84 and S87 phosphosites of AbfR1 are less conserved among the OxsR homologs than the OxsR C24, we 
find OxsR to have residues (Y88 and T91) analogous to the sites of AbfR1 phosphorylation suggesting PTM crosstalk between a thiol-switch and phosphorylation.

In this work we revealed the requirement of OxsR when $H$. volcanii is exposed to oxidative stress. This work nicely complements previous findings of RosR function in the haloarchaeal oxidative stress response (61-63), as the mode of DNA binding and mechanism of sensing oxidant appear quite distinct between OxsR and RosR (the latter yet to be determined). OxsR binding regions that were mapped by using a genome-wide ChIP-seq approach provide the biological roles not only as an activator for genes involved in amino acid and thiol transfer but a repressor for DNA repair system (Figure 8). The conserved DNA motif (CGGnCGnGCG) and cysteine residue are mainly contributed to the OxsR binding, however, other factors that interact with OxsR to find/bind to target DNA regions would be remained to be discovered. Overall, this work supports an emerging principle that OxsR which is widespread in most archaeal phyla plays a pivotal role against oxidative stress.

\section{Materials and Methods}

Materials. Biochemicals and analytical-grade inorganic chemicals were purchased from Fisher Scientific (Atlanta, GA), Bio-Rad (Hercules, CA) and Sigma-Aldrich (St. Louis, MO). Desalted oligonucleotides were from Integrated DNA Technologies (Coralville, IA). DNA polymerases and restriction enzymes were from New England Biolabs (Ipswich, MA) and Clontech Laboratories, Inc. (Mountain View, CA). Hi-Lo DNA standards were from Minnesota Molecular, Inc. (Minneapolis, MN).

Strains, media and growth conditions. Details of strains, plasmids, and primer sequences used in this study are listed in Table S4. E. coli cultures were grown at $37^{\circ} \mathrm{C}$ in Luria-Bertani (LB) medium. $H$. volcanii strains were grown at $42{ }^{\circ} \mathrm{C}$ in ATCC974 complex medium or glycerol 
minimal medium (GMM) as previously described (77). Liquid cultures were grown with rotary agitation at $200 \mathrm{rpm}$. Media was supplemented with 1.5\% agar for plates and with novobiocin $\left(\mathrm{Nv}, 0.2 \mu \mathrm{g} \cdot \mathrm{ml}^{-1}\right)$ or ampicillin $\left(\mathrm{Ap}, 100 \mu \mathrm{g} \cdot \mathrm{ml}^{-1}\right)$ as needed. Manipulation of $H$. volcanii strains and DNA was as described by the Halohandbook (http://www.haloarchaea.com/resources/halohandbook/). Plasmids were generated in E. coli TOP10 and then transferred to E. coli GM2163 prior to transformation of $H$. volcanii. Strain and plasmid fidelity was confirmed by Sanger DNA sequencing (Eton Bioscience, Inc., San Diego, CA).

Generation of $\boldsymbol{H}$. volcanii mutant strains. The $H$. volcanii $\Delta$ oxsR mutant (SH125) was generated using $H$. volcanii $\mathrm{H} 26$ as the parent by a pyrE2-based pop-in/pop-out deletion method (48). The pre-deletion plasmid pJAM3380 was generated by ligation of a DNA fragment carrying oxs $R$ and 5' and 3' flanking regions (about 600 bp each) into the HindllI to Xbal sites of plasmid pTA131. The oxs $R$ region carried on pJAM3380 was generated by PCR using primer pair $1 / 2$ and $H$. volcanii $\mathrm{H} 26$ genomic DNA as a template. Deletion plasmid pJAM3381 was constructed by inverse PCR using primer pair 3/4 and plasmid pJAM3380 as a template. Primer pairs used to screen for the SH125 mutant included 1/2, 3/4, and 5/6 (primers outside the deletion plasmid). Plasmid pJAM3388 was created by ligating a DNA fragment carrying oxs $R$ into the Ndel and Kpnl sites of pJAM809. The oxsR region carried on pJAM3388 was generated by PCR using primer pair 5/6 and $H$. volcanii $\mathrm{H} 26$ genomic DNA as a template. This plasmid and the empty vector (pJAM202c) were transformed into the $\Delta$ oxsR mutant $(\mathrm{SH} 125)$ for complementation assay. A similar pop-in/pop-out strategy was used to generate the other $H$. volcanii strains including SH126, PM057, PM058, PM059, and PM012. Plasmid pJAM3389 used to integrate the oxsR::HA coding sequence into the oxs $R$ locus was generated by inverse PCR using primer pair 7/8 and plasmid pJAM3380 a template. Strain SH126 was screened using primer pair 9/10. For site-directed mutagenesis to generate pJAM3901 (that encodes 
OxsR C24A with C-terminal HA tag), plasmid pJAM3389 and overlapping primer pair 11/12 were used to amplify a linear plasmid by PCR that was Dpnl treated to remove template and ligated using a KLD Enzyme Mix (NEB). For deletion of hvo_1043, the hvo_1043 region carried on pJAM3919 was generated by PCR using primer pair 27/28 and $H$. volcanii H26 genomic DNA as a template. Deletion plasmid pJAM3920 was constructed by inverse PCR using primer pair 29/30 and plasmid pJAM3919 as a template. Primer pairs used to screen for mutants included 27/28 (primers outside the deletion plasmid). The deletions of the CG-rich motif 5 ' of hvo_1043 were performed in a similar matter but used different primers. To generate the $24 \mathrm{bp}$ deletion, primer pair 31/32 was used for inverse PCR with pJAM3919 as template. For the 12 bp deletion of the CG repeat, primer pair 33/34 was used for inverse PCR with pJAM3919 as template. All constructed plasmids were verified by the Sanger DNA sequencing method (Eton Bioscience, Research Triangle Park, NC). The SH125 ( $\Delta$ oxsR) mutant was also confirmed by comparison to the H26 parent using the breseq pipeline (96) for whole genome sequencing (Table S1).

Hypochlorite stress growth assay. For hypochlorite stress growth assays, the following method was used. The $H$. volcanii strains were streaked from $-80^{\circ} \mathrm{C}$ glycerol stocks onto glycerol minimal medium (GMM) plates and incubated for 5 days in a closed plastic zippered bag at $42{ }^{\circ} \mathrm{C}$. Five isolated colonies were inoculated into $50 \mathrm{ml}$ of GMM in $250 \mathrm{ml}$ Erlenmeyer flasks. Cells were grown to late log phase $\left(\mathrm{OD}_{600}\right.$ of $\left.0.85-0.95\right)$ at $42{ }^{\circ} \mathrm{C}(200 \mathrm{rpm}$, rotary shaking). Cells were diluted with fresh $\mathrm{GMM}$ to a $\mathrm{OD}_{600}$ of 0.1 unit in $95 \mathrm{ml}$ final volume. Aliquots $(5 \mathrm{~mL})$ of this cell suspension were transferred into 12 loosely capped $13 \times 100 \mathrm{~mm}$ culture tubes per strain type and incubated for $10 \mathrm{~h}$ at $42^{\circ} \mathrm{C}$ with aeration using a mini rotator (Glas-Col from Terre Haute in the USA) at a max percent speed setting of 50 . Once cells reached log phase (OD600 of $0.4-0.6$ ), half of the 12 tubes were randomly selected for supplementation with or without $5 \mathrm{mM} \mathrm{NaOCl}$ (sodium hypochlorite reagent grade, available chlorine $10-15 \%$, Sigma- 
Aldrich, \#425044-250mL), which forms hypochlorite in solution. The tubes were returned to the mini rotator and cell growth was monitored for 10 days at OD600 using a Spectronic 20+ spectrophotometer (ThermoSpectronic, Filter: 600-950nm). The experiment was determined to be reproducible ( $n=12$ total; 6 tubes per strain plus condition for each of 2 experiments). Initial analysis by circular rotary shaking yielded variable results, most likely due to the microaerobic conditions which promoted extensive incubation times after exposure to hypochlorite.

Quantitative real-time reverse transcriptase PCR (qRT-PCR) analysis. $H$. volcanii strains for qRT-PCR analysis were streaked from $-80^{\circ} \mathrm{C}$ glycerol stocks onto defined minimal media (GMM) and incubated for five days in a sealed plastic zippered bag at $42^{\circ} \mathrm{C}$. Isolated colonies were inoculated into $20 \mathrm{ml}$ of GMM in $125 \mathrm{ml}$ Erlenmeyer flasks. Cells were grown to early log phase $\left(\mathrm{OD}_{600}\right.$ of $\left.0.3-0.5\right)$ at $42^{\circ} \mathrm{C}(200 \mathrm{rpm})$. For each strain, aliquots (1 ml) of the cell culture (20 $\mathrm{ml}$ ) were transferred to $1.5 \mathrm{ml}$ microcentrifuge tubes (RNase-free), and each sample was exposed to $2.5 \mathrm{mM}$ of $\mathrm{NaOCl}$ for different times $(5,10,15$, and $20 \mathrm{~min})$ at $42^{\circ} \mathrm{C}(200 \mathrm{rpm})$. A mock control was included for comparison of each time point. Total RNA was isolated from cells using TRI Reagent (\#T9424) according to the supplier (Sigma-Aldrich). TURBO DNA-free ${ }^{\text {TM }}$ Kit (AM1907) was used to remove contaminating DNA from the RNA samples according to the supplier's recommendations (Invitrogen). Only RNA samples with DNA below the limit of PCR detection were further processed. RNA integrity was confirmed by mixing samples $1: 1$ in $2 X$ RNA loading dye (B0363S; New England Biolabs) and separating by $0.8 \%$ (w/v) agarose gel electrophoresis in 1X TBE. Only RNA (2 ng) samples with no apparent degradation served as the template for qRT-PCR in $20 \mu \mathrm{l}$ reactions. The Luna Universal One-Step RT-qPCR kit (E3005L) was used for qRT-PCR analysis following the protocol described by the supplier (NEB) by using a CFX96 real-time C1000 thermal cycler (Bio-Rad). The reverse-transcription was performed under conditions of $55^{\circ} \mathrm{C}$ for $10 \mathrm{~min}$. The quantitative real-time PCR was performed under conditions of 40 cycles at $95^{\circ} \mathrm{C}$ for $1 \mathrm{~min}, 95^{\circ} \mathrm{C}$ for $10 \mathrm{~s}$, and $56^{\circ} \mathrm{C}$ for $15 \mathrm{~s}$. An 
extension of $60^{\circ} \mathrm{C}$ for $30 \mathrm{~s}$ was performed followed by determination of the melting curve under conditions of $95^{\circ} \mathrm{C}$ for $10 \mathrm{~s}$ and increase in temperature from $60^{\circ} \mathrm{C}$ to $95^{\circ} \mathrm{C}$ for $5 \mathrm{~s}$ each. A single peak revealed by the melting curve indicated a single product. The internal standard HVO_1015 was used to normalize the target mRNA levels, based on finding its transcript levels were unperturbed by $\mathrm{HOCl}$ stress. Genomic DNA served as the template to test different primer pairs for PCR efficiency. Primers with PCR efficiency between $95 \%$ and $105 \%$ are listed in Table S4. For the analysis for qRT-PCR, fold-ratios of relative-fold change in gene expression were calculated using the $2^{-\Delta \Delta C t}$ method (Livak \& Schmittgen, 2001) For the statistical analysis, the student's t-test was conducted to compare the mean of gene expression between the H26 parent and $\Delta$ oxsR mutant strain (SH125). For the qRT-PCR analysis of the $\Delta h v o \_1043$ mutant (PM057), strains with deletions of the OxsR binding motif identified 5' of the promoter region of hvo_1043 (PM058 and PM059), and the oxsR-HA C24A integrant strain (PM012), the same method was used as explained above, except no time-course was performed. The strains were exposed to $2.5 \mathrm{mM} \mathrm{NaOCl}$ for $15 \mathrm{~min}$, and a mock control was included for each strain. All experiments were performed in biological duplicate or quadlets and technical triplicate.

Preparation for ChIP-sequencing and data analysis. Four single colonies of SH126 (oxsR$H A$ integrant) and two colonies of $\mathrm{H} 26$ were inoculated in 5-mL GMM and grown aerobically at $42^{\circ} \mathrm{C}$ to early stationary phase to synchronize growth phase (OD600nm, $\left.\sim 1.0\right)$ with shaking (200 rpm). Cells were transferred to fresh 100-mL GMM, and $2.5 \mathrm{mM} \mathrm{NaOCl}$ was added for 20 min when cells reached log phase (OD600nm, 0.3 0.5) for the oxidative stress group. A mock control was included for comparison. ChIP-seq samples were prepared as the previous method with modifications (97). Briefly, to crosslink, $37 \%$ formaldehyde was added to the culture at the final concentration of $1 \%$ and the cell culture was incubated on a rocking platform for 20 min at room temperature. A final concentration of $0.125 \mathrm{M}$ glycine was added to stop the crosslinking reaction and the whole cells were washed three times with cold basal salts buffer followed by 
storage at $-80^{\circ} \mathrm{C}$ until sonication. The cell pellet was thawed and resuspended in $800 \mu$ lysis buffer (50 mM HEPES, 140 mM NaCl, 1 mM EDTA, 1\% (v/v) Triton X-100, 0.1\% (w/v) sodium deoxycholate, $\mathrm{pH}$ 7.5) containing protease inhibitor cocktail (Thermo Scientific) to shear DNA by Bioruptor 300 sonication system (Diagenode) with 15 cycles of $30 \mathrm{~s}$ on and $90 \mathrm{~s}$ off at high magnitude. The sheared DNA was monitored to confirm the genomic DNA was a smear between $200-800$ bp with the highest concentration of fragments $\sim 500$ bp by $1.2 \%(w / v)$ agarose gel electrophoresis. For immunoprecipitation, the sheared DNA was immediately incubated overnight with the complex anti-HA tag antibody (Abcam, ab9110) and Dynabeads protein A (Invitrogen) at $4^{\circ} \mathrm{C}$. Enriched DNA/OxsR complexes were eluted by adding $50 \mu \mathrm{l}$ elution buffer (50 mM Tris, $10 \mathrm{mM}$ EDTA, $1 \%$ SDS (w/v), $\mathrm{pH} 8.0$ ) and incubation at $65^{\circ} \mathrm{C}$ for 10 min. Reverse crosslinking was performed by incubating in TE/SDS (10 mM Tris, 1 mM EDTA, $1 \%$ SDS) overnight at $65^{\circ} \mathrm{C}$. DNA, of which the RNA was removed, was subsequently extracted by a phenol-chloroform method. Library preparation and deep sequencing were carried out at Duke sequencing core. Sequencing reads were trimmed and controlled their quality (Phred score $>30$ ) by TrimGalore! wrapper pipeline (https://github.com/FelixKrueger/TrimGalore) with default parameters. The preprocessed reads were mapped (alignment rate > 95\%) using Bowtie2 (98) to the $H$. volcanii DS2 reference genome (https://www.ncbi.nlm.nih.gov/genome/1149?genome_assembly_id=170797). The mapped reads were then sorted and indexed by Samtools (99). Peaks (cutoff, Qval < 0.05) were called by MOSAiCS (100) and checked for quality by ChIPQC (101). DiffBind was used to identify significant peaks that were present at least three of four biological replicates (102), and ChIPseeker was used to annotate the peaks (see the peak information in Table S2) (103). Peak heights reported represent the mean of the ratio of read counts in the IP sample vs input control. Integrative genomics viewer (IGV) was used for the manual evaluation of peak height and peak location, as well as for the data visualization (104). For all gene lists harboring peaks in their upstream coding region, a functional enrichment was performed with arCOG categories (67) 
based on the hypergeometric distribution test as described previously $(105,106)$. Code associated with this ChIP-seq analysis are freely available at https://github.com/amyschmid/OxsR_ChIP_WGS.

Immunoprecipitation of OxsR-HA with and without the C24A mutation. Hfx. volcanii strains H26, SH126 (H26 oxsR:HA integrant) and PM012 (H26 oxsR:HA C24A integrant) were inoculated from ATCC 974 plates to glycerol minimal medium (GMM) (3 ml in $13 \times 100 \mathrm{~mm}$ tubes) and grown to late log phase (OD600, 0.8-1.0). Cells were diluted to an OD600 of 0.03 into fresh GMM (25 ml in $250 \mathrm{ml}$ Erlenmeyer flask) and grown to log phase (OD600, 0.8-1.0). Cells were transferred to fresh GMM (200 ml cultures in $1 \mathrm{~L}$ Erlenmeyer flask) and grown to stationary phase $(\mathrm{OD} 600,1.6)$. Cells were harvested by centrifugation $(10,000 \times g$ for $30 \mathrm{~min}, 4$ oC). Cell pellets were washed with $150 \mathrm{ml} 20 \%$ salt water (SW, where $20 \%$ is composed of 2.46 M NaCl, $88 \mathrm{mM} \mathrm{MgCl}_{2}, 142 \mathrm{mM} \mathrm{MgSO}_{4}, 56.3 \mathrm{mM} \mathrm{KCl}, 42 \mathrm{mM}$ Tris-Cl, pH 7.5) by centrifugation $(10,000 \times g$ for $20 \mathrm{~min}, 4 \circ \mathrm{C})$. Cell pellets were stored at $-80^{\circ} \mathrm{C}$ for 2 days before used. Cell pellets were resuspended in $0.8 \mathrm{ml}$ of $0.2 \%$ (w/v) SDS in lysis buffer composed of 50 mM HEPES, 2 mM EDTA, $150 \mathrm{mM} \mathrm{NaCl,} 1 \%$ (v/v) Triton X-100, $0.1 \%$ (w/v) sodium deoxycholate, $\mathrm{pH}$ 8.0. Throughout the immunoprecipitation experiment, the buffers were maintained on ice and supplemented with protease inhibitor cocktail according to supplier (Sigma). The resuspended cells were incubated on ice for $15 \mathrm{~min}$ and subsequently sonicated with an aspiration probe for 10 cycles (10 pulses, $0.5 \mathrm{~s}$ on, $0.5 \mathrm{~s}$ off at $30 \%$ amplitude) (Sonic Dismembrator Model 500 fitted with a Branson model 102C aspiration probe, Fisher Scientific and Branson Ultrasonics, Danbury, CT) with ice-slurry incubations of at least 1 min between cycles. The sonicated samples were centrifuged (16873 $\times g$ for $\left.30 \mathrm{~min}, 4^{\circ} \mathrm{C}\right)$. The supernatant was transferred to a $15 \mathrm{ml}$ Falcon tube (Fisher Scientific), supplemented with $1 \mu \mathrm{g}$ of $\alpha-\mathrm{HA}$ antibody (ChIP Grade, product \# ab9110, Abcam, Cambridge, MA) in $3 \mathrm{ml}$ ice-cold ChIP lysis buffer and incubated for $4 \mathrm{~h}$ at $4{ }^{\circ} \mathrm{C}$ with rocking. During this time, Protein A Dynabeads $(50 \mu l$, 
Invitrogen) were prewashed two times in a $2 \mathrm{ml}$ microcentrifuge tube (natural color) with $1 \mathrm{ml} 1$ × phosphate buffered saline (PBS) at pH 7.4 (containing $8.0 \mathrm{~g} \mathrm{NaCl}, 0.2 \mathrm{~g} \mathrm{KCl}, 1.44 \mathrm{~g} \mathrm{Na}_{2} \mathrm{HPO}_{4}$, and $0.24 \mathrm{~g} \mathrm{KH}_{2} \mathrm{PO}_{4}$ per liter) (where the beads were washed by application of slurry to a magnet and removal of supernatant by aspiration). Beads were blocked by addition of $400 \mu \mathrm{l}$ BSA in PBS buffer $\left(1 \mathrm{mg} \cdot \mathrm{ml}^{-1}\right)$. The BSA-bead slurry was added to the pre-incubated sample and further incubated overnight (rocking at $4{ }^{\circ} \mathrm{C}$ ). After incubation, the supernatant was removed from the beads (via magnet and aspiration), and the beads were resuspended in ice-cold lysis buffer $(1 \mathrm{ml})$. The bead-slurry was transferred to a fresh $2 \mathrm{ml}$ tube, and the supernatant was removed by application to a magnet and aspiration. This wash step was repeated for a total of two times and followed by subsequent washing steps that were each repeated twice with the following buffers: wash buffer 1 (lysis buffer with $150 \mathrm{mM} \mathrm{NaCl}$ ), wash buffer 2 [10 mM Tris-Cl, 2 mM EDTA, 25 mM LiCl, $1 \%$ (w/v) Nonidet P-40, $1 \%$ (w/v) sodium deoxycholate, $\mathrm{pH}$ 8.0] and TE buffer (10 mM Tris-Cl, 1 mM EDTA, pH 8.0) respectively. After washing, the beads were resuspended in $100 \mu \mathrm{l}$ 2x SDS reducing buffer (125 mM Tris- $\mathrm{HCl}, \mathrm{pH} 6.8,20 \%$ glycerol, 4\% SDS, $0.1 \%$ bromophenol blue and $5 \% \beta$-mercaptoethanol) and boiled for $10 \mathrm{~min}$. The samples were centrifuged $(5,000 \times g$ for $5 \mathrm{~min}$, room temperature). The supernatant was stored in a fresh $2 \mathrm{ml}$ microcentrifuge tube and analyzed by immunoblotting. The remaining supernatant was stored at $-20^{\circ} \mathrm{C}$ for future use.

Immunoblotting (western) analysis. Immunoprecipitants (10 $\mu$ l per lane) were separated by reducing $15 \%$ SDS-PAGE. Proteins were transferred to PVDF membrane at $4^{\circ} \mathrm{C}$ for $14.5 \mathrm{~h}$ at 30 $\mathrm{V}$ using the mini trans-blot module in transblot buffer (10 mM MES buffer $\mathrm{pH} 6$ and 10\% (v/v) methanol) according to the supplier's instructions (Bio-Rad). The membrane was removed from the cassette, and the location of the gel and protein standards were marked on the membrane using a pencil. The membrane was placed upright in an 18 by $10 \mathrm{~cm}$ plastic container and rinsed with $30 \mathrm{ml}$ of $1 \times$ TBST (50 mM Tris- $\mathrm{Cl}, \mathrm{pH} 7.5,150 \mathrm{mM} \mathrm{NaCl}$, and $0.5 \mathrm{ml} / \mathrm{L}$ Tween 20) for 
$30 \mathrm{~min}$. The membrane was soaked with $100 \%$ methanol and dried for $1 \mathrm{~h}$ at room temperature under laminar air flow. The membrane was reactivated with $100 \%$ methanol, washed briefly with 1X TBST three times, and blocked for $3 \mathrm{~h}$ at $4^{\circ} \mathrm{C}$ in $60 \mathrm{ml}$ blocking buffer composed of TBST buffer supplemented with 5\% (w/v) BSA (Sigma Life Science). During the blocking stage, the membrane was gently rocked using the Lab-Line Rocker on medium (5 or 6 ) setting. The blocking solution was replaced with a solution of anti-HA tag horseradish peroxidase (HRP) antibody (ab1190) diluted to 1:20,000 in $60 \mathrm{ml}$ of the blocking buffer. The membrane was incubated with gentle rocking for $1 \mathrm{~h}$ at $4^{\circ} \mathrm{C}$. The membrane was rinsed for $1.1 \mathrm{~h}$ at room temperature with $50 \mathrm{ml}$ TBST buffer seven times using the high setting of the rocker. The HRP signal of the antibody: protein complexes was visualized on the PVDF membrane by chemiluminescence using $2 \mathrm{ml}$ of Amersham ECL Prime from CGE Healthcare Life Sciences and exposure to the iBright FL1500 Imaging System (A44241).

Computational prediction of OxsR-binding DNA motifs. DNA fragments identified by ChIPseq to be bound to OxsR were tested for common DNA motif(s) using the MEME Suite v. 4.12.0 (70). Sequences were input into the de novo motif detection mode of MEME-MAST with the following parameters: any number of repeats, max width of $24 \mathrm{bp}$, and 3 output motifs. Two DNA sequence sets were used as input. The first set, which did not generate DNA motifs of high significance, included all DNA sequences bound by OxsR. The second set, which identified the

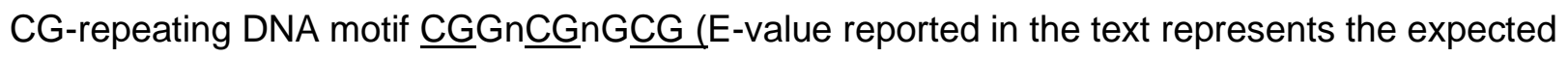
number of sequences in a random database of the same size that would match the motif), was a subset of the OxsR-bound DNA sequences and was supplemented with analogous intergenic regions from related haloarchaeal genomes. These related regions were retrieved by comparing the deduced protein sequences of the flanking genes by Basic Local Alignment Search Tool using BlastP (protein-protein BLAST) (107). The DNA sequences 5' of the genes encoding these homologs were retrieved using the graphics tool within NCBI nucleotide portal 
(https://www.ncbi.nlm.nih.gov/nuccore/). DNA motifs identified in this manner were compared to shuffled sequences to determine significance. DNA motifs found to be significant (based on uniqueness to the unshuffled sequences) were input into the FIMO algorithm (71) to scan the Hfx. volcanii genome database (db/upstream_Prokaryotic_Haloferax_volcanii_DS2_uid46845_2018-06-11.fna) of 4073 sequences and 1,428,983 residues. The DNA sequences used to identify the $\underline{\mathrm{CG}} \underline{\mathrm{G}} \underline{\mathrm{G}} \mathrm{nG} \underline{\mathrm{CG}}$ motif, and the FIMO output of the genome scanning are provided in Table S2.

Computational prediction of OxsR 3D-structure. Protein sequences from UniProtKB accession numbers D4GY41 and D4GXQ1 were used to model 3D-structures of OxsR and HVO_1360, respectively. The Phyre2 web portal (72) was used to predict structure by fold recognition threading. RoseTTAfold (73) was used for de novo structure prediction. The 3Dmodels were visualized and compared using ChimeraX (108).

Data availability. The ChIP-seq data discussed in this publication have been deposited in NCBI's Gene Expression Omnibus $(109,110)$ and are accessible through GEO Series accession number GSE196894 (https://www.ncbi.nlm.nih.gov/geo/query/acc.cgi?acc= GSE196894). The whole genomic sequence (WGS) data for the $\Delta$ oxsR mutant (SH125) and oxsR-HA integrant (SH126) are deposited with accession number PRJNA806939 in the Sequence Read Archive (SRA)(111). Code and input data associated with the ChIP-seq analysis are freely available for download via GitHub:

https://github.com/amyschmid/OxsR_ChIP_WGS.

Acknowledgments. Funds awarded to JM and AS to develop systems biology tools were through the Bilateral NSF/BIO-BBSRC program (NSF 1642283). Funds awarded to JM to determine redox regulation in archaea were through the U.S. Department of Energy, Office of Basic Energy Sciences, Division of Chemical Sciences, Geosciences and Biosciences, Physical 
Biosciences Program (DOE DE-FG02-05ER15650). Funds awarded to JM to provide evolutionary insight in biological systems were through the National Institutes of Health (NIH 5R01GM057498). Funds to AKS to understand mechanisms of stress response affecting cell growth and gene network evolution were provided by the National Science Foundation (NSF 1651117 and 1936024).

Author contributions. SH, PM, AS and JM designed the research experiments, analyzed the data, and wrote the paper. $\mathrm{PM}, \mathrm{SH}$ and $\mathrm{RO}$ generated the genetic constructs unique to this study. PM and LK performed the qRT-PCR analysis. SH, PM, AS, and JM analyzed the global datasets. AN, EW, and RC provided insight into OxsR disulfide bond formation. All authors performed research experiments associated with this paper and have read and approved of the paper. The authors do not have a conflict of interest to declare.

\section{References}

1. Fasnacht M, Polacek N. 2021. Oxidative stress in bacteria and the central dogma of molecular biology. Front Mol Biosci 8:671037.

2. Georgiou G. 2002. How to flip the (redox) switch. Cell 111:607-10.

3. Karr EAI, C.E.; Trinh, V.; Peeters, E. 2017. Transcription factor mediated gene regulation in Archaea, p 27-69. In Clouet-d'Orval B (ed), RNA Metabolism and Gene Expression in Archaea, vol 32. Springer International Publishing.

4. Hillion M, Antelmann H. 2015. Thiol-based redox switches in prokaryotes. Biol Chem $396: 415-44$.

5. Antelmann H, Helmann JD. 2011. Thiol-based redox switches and gene regulation. Antioxid Redox Signal 14:1049-63.

6. Drazic A, Winter J. 2014. The physiological role of reversible methionine oxidation. Biochim Biophys Acta 1844:1367-1382. 
7. Vazquez-Torres A. 2012. Redox active thiol sensors of oxidative and nitrosative stress. Antioxid Redox Signal 17:1201-14.

8. Crack JC, Green J, Thomson AJ, Le Brun NE. 2014. Iron-sulfur clusters as biological sensors: the chemistry of reactions with molecular oxygen and nitric oxide. Acc Chem Res 47:3196-205.

9. Lemmens L, Maklad HR, Bervoets I, Peeters E. 2019. Transcription regulators in Archaea: homologies and differences with bacterial regulators. J Mol Biol.

10. Wenck BR, Santangelo TJ. 2020. Archaeal transcription. Transcription 11:199-210.

11. Fuangthong M, Atichartpongkul S, Mongkolsuk S, Helmann JD. 2001. OhrR is a repressor of ohrA, a key organic hydroperoxide resistance determinant in Bacillus subtilis. J Bacteriol 183:4134-41.

12. Lee JW, Soonsanga S, Helmann JD. 2007. A complex thiolate switch regulates the Bacillus subtilis organic peroxide sensor OhrR. Proc Natl Acad Sci U S A 104:8743-8.

13. Newberry KJ, Fuangthong M, Panmanee W, Mongkolsuk S, Brennan RG. 2007. Structural mechanism of organic hydroperoxide induction of the transcription regulator OhrR. Mol Cell 28:652-64.

14. Sun F, Ding Y, Ji Q, Liang Z, Deng X, Wong CC, Yi C, Zhang L, Xie S, Alvarez S, Hicks LM, Luo C, Jiang H, Lan L, He C. 2012. Protein cysteine phosphorylation of SarA/MgrA family transcriptional regulators mediates bacterial virulence and antibiotic resistance. Proc Natl Acad Sci U S A 109:15461-6.

15. Chi BK, Gronau K, Mäder U, Hessling B, Becher D, Antelmann H. 2011. Sbacillithiolation protects against hypochlorite stress in Bacillus subtilis as revealed by transcriptomics and redox proteomics. Mol Cell Proteomics 10:M111.009506.

16. Loi VV, Busche T, Tedin K, Bernhardt J, Wollenhaupt J, Huyen NTT, Weise C, Kalinowski J, Wahl MC, Fulde M, Antelmann H. 2018. Redox-sensing under hypochlorite 
stress and infection conditions by the Rrf2-family repressor HypR in Staphylococcus aureus. Antioxid Redox Signal 29:615-636.

17. Lee SJ, Lee IG, Lee KY, Kim DG, Eun HJ, Yoon HJ, Chae S, Song SH, Kang SO, Seo MD, Kim HS, Park SJ, Lee BJ. 2016. Two distinct mechanisms of transcriptional regulation by the redox sensor YodB. Proc Natl Acad Sci U S A 113:E5202-11.

18. Ji Q, Zhang L, Jones MB, Sun F, Deng X, Liang $H$, Cho H, Brugarolas $P$, Gao YN, Peterson SN, Lan L, Bae T, He C. 2013. Molecular mechanism of quinone signaling mediated through S-quinonization of a YodB family repressor QsrR. Proc Natl Acad Sci U S A 110:5010-5.

19. Brugarolas P, Movahedzadeh F, Wang Y, Zhang N, Bartek IL, Gao YN, Voskuil MI, Franzblau SG, He C. 2012. The oxidation-sensing regulator (MosR) is a new redoxdependent transcription factor in Mycobacterium tuberculosis. J Biol Chem 287:3770312.

20. Chen PR, Nishida S, Poor CB, Cheng A, Bae T, Kuechenmeister L, Dunman PM, Missiakas D, He C. 2009. A new oxidative sensing and regulation pathway mediated by the MgrA homologue SarZ in Staphylococcus aureus. Mol Microbiol 71:198-211.

21. Zheng M, Aslund F, Storz G. 1998. Activation of the OxyR transcription factor by reversible disulfide bond formation. Science 279:1718-21.

22. Zheng M, Wang X, Doan B, Lewis KA, Schneider TD, Storz G. 2001. Computationdirected identification of OxyR DNA binding sites in Escherichia coli. J Bacteriol $183: 4571-9$

23. Teramoto $\mathrm{H}$, Inui M, Yukawa H. 2013. OxyR acts as a transcriptional repressor of hydrogen peroxide-inducible antioxidant genes in Corynebacterium glutamicum R. FEBS J 280:3298-312.

24. Touati D. 2000. Sensing and protecting against superoxide stress in Escherichia coli-how many ways are there to trigger soxRS response? Redox Rep 5:287-93. 
25. Demple B, Hidalgo E, Ding H. 1999. Transcriptional regulation via redox-sensitive ironsulphur centres in an oxidative stress response. Biochem Soc Symp 64:119-28.

26. Khoroshilova N, Popescu C, Münck E, Beinert H, Kiley PJ. 1997. Iron-sulfur cluster disassembly in the FNR protein of Escherichia coli by $\mathrm{O}_{2}$ : [4Fe-4S] to [2Fe-2S] conversion with loss of biological activity. Proc Natl Acad Sci U S A 94:6087-92.

27. Hidese R, Yamashita K, Kawazuma K, Kanai T, Atomi H, Imanaka T, Fujiwara S. 2017. Gene regulation of two ferredoxin:NADP ${ }^{+}$oxidoreductases by the redox-responsive regulator SurR in Thermococcus kodakarensis. Extremophiles 21:903-917.

28. Lim JK, Jung HC, Kang SG, Lee HS. 2017. Redox regulation of SurR by protein disulfide oxidoreductase in Thermococcus onnurineus NA1. Extremophiles 21:491-498.

29. Yang H, Lipscomb GL, Keese AM, Schut GJ, Thomm M, Adams MW, Wang BC, Scott RA. 2010. SurR regulates hydrogen production in Pyrococcus furiosus by a sulfurdependent redox switch. Mol Microbiol 77:1111-22.

30. Lipscomb GL, Schut GJ, Scott RA, Adams MWW. 2017. SurR is a master regulator of the primary electron flow pathways in the order Thermococcales. Mol Microbiol 104:869881.

31. Karr EA. 2010. The methanogen-specific transcription factor MsvR regulates the fpaArlp-rub oxidative stress operon adjacent to $m s v R$ in Methanothermobacter thermautotrophicus. J Bacteriol 192:5914-22.

32. Sheehan R, McCarver AC, Isom CE, Karr EA, Lessner DJ. 2015. The Methanosarcina acetivorans thioredoxin system activates DNA binding of the redox-sensitive transcriptional regulator MsvR. J Ind Microbiol Biotechnol 42:965-9.

33. Isom CE, Turner JL, Lessner DJ, Karr EA. 2013. Redox-sensitive DNA binding by homodimeric Methanosarcina acetivorans MsvR is modulated by cysteine residues. BMC Microbiol 13:163. 
34. Maruyama H, Shin M, Oda T, Matsumi R, Ohniwa RL, Itoh T, Shirahige K, Imanaka T, Atomi H, Yoshimura SH, Takeyasu K. 2011. Histone and TK0471/TrmBL2 form a novel heterogeneous genome architecture in the hyperthermophilic archaeon Thermococcus kodakarensis. Mol Biol Cell 22:386-98.

35. Kim M, Park S, Lee SJ. 2016. Global transcriptional regulator TrmB family members in prokaryotes. J Microbiol 54:639-45.

36. Wagner M, Wagner A, Ma X, Kort JC, Ghosh A, Rauch B, Siebers B, Albers SV. 2014. Investigation of the malE promoter and MalR, a positive regulator of the maltose regulon, for an improved expression system in Sulfolobus acidocaldarius. Appl Environ Microbiol 80:1072-81.

37. Lee SJ, Surma M, Seitz S, Hausner W, Thomm M, Boos W. 2007. Characterization of the TrmB-like protein, PF0124, a TGM-recognizing global transcriptional regulator of the hyperthermophilic archaeon Pyrococcus furiosus. Mol Microbiol 65:305-18.

38. Reichelt R, Gindner A, Thomm M, Hausner W. 2016. Genome-wide binding analysis of the transcriptional regulator TrmBL1 in Pyrococcus furiosus. BMC Genomics 17:40.

39. Kanai T, Akerboom J, Takedomi S, van de Werken HJ, Blombach F, van der Oost J, Murakami T, Atomi H, Imanaka T. 2007. A global transcriptional regulator in Thermococcus kodakaraensis controls the expression levels of both glycolytic and gluconeogenic enzyme-encoding genes. J Biol Chem 282:33659-70.

40. Schmid AK, Reiss DJ, Pan M, Koide T, Baliga NS. 2009. A single transcription factor regulates evolutionarily diverse but functionally linked metabolic pathways in response to nutrient availability. Mol Syst Biol 5:282.

41. Todor H, Dulmage K, Gillum N, Bain JR, Muehlbauer MJ, Schmid AK. 2014. A transcription factor links growth rate and metabolism in the hypersaline adapted archaeon Halobacterium salinarum. Mol Microbiol 93:1172-82. 
42. Lee SJ, Moulakakis C, Koning SM, Hausner W, Thomm M, Boos W. 2005. TrmB, a sugar sensing regulator of $\mathrm{ABC}$ transporter genes in Pyrococcus furiosus exhibits dual promoter specificity and is controlled by different inducers. Mol Microbiol 57:1797-807.

43. Lee SJ, Engelmann A, Horlacher R, Qu Q, Vierke G, Hebbeln C, Thomm M, Boos W. 2003. TrmB, a sugar-specific transcriptional regulator of the trehalose/maltose ABC transporter from the hyperthermophilic archaeon Thermococcus litoralis. J Biol Chem 278:983-90.

44. Krug M, Lee SJ, Boos W, Diederichs K, Welte W. 2013. The three-dimensional structure of $\operatorname{TrmB}$, a transcriptional regulator of dual function in the hyperthermophilic archaeon Pyrococcus furiosus in complex with sucrose. Protein Sci 22:800-8.

45. Reichlen MJ, Vepachedu VR, Murakami KS, Ferry JG. 2012. MreA functions in the global regulation of methanogenic pathways in Methanosarcina acetivorans. MBio 3:e00189-12.

46. Leyn SA, Rodionova IA, Li X, Rodionov DA. 2015. Novel transcriptional regulons for autotrophic cycle genes in Crenarchaeota. J Bacteriol 197:2383-91.

47. Wierer S, Daldrop P, Ud Din Ahmad M, Boos W, Drescher M, Welte W, Seidel R. 2016. TrmBL2 from Pyrococcus furiosus interacts both with double-stranded and singlestranded DNA. PLoS One 11:e0156098.

48. Mormile MR, Biesen MA, Gutierrez MC, Ventosa A, Pavlovich JB, Onstott TC, Fredrickson JK. 2003. Isolation of Halobacterium salinarum retrieved directly from halite brine inclusions. Environ Microbiol 5:1094-102.

49. Oren A. 2002. Diversity of halophilic microorganisms: environments, phylogeny, physiology, and applications. J Ind Microbiol Biotechnol 28:56-63.

50. Arrage AA, Phelps TJ, Benoit RE, White DC. 1993. Survival of subsurface microorganisms exposed to UV radiation and hydrogen peroxide. Appl Environ Microbiol $59: 3545-50$. 
51. Jones DL, Baxter BK. 2017. DNA repair and photoprotection: Mechanisms of overcoming environmental ultraviolet radiation exposure in halophilic archaea. Front Microbiol 8:1882.

52. Oren A. 2008. Microbial life at high salt concentrations: phylogenetic and metabolic diversity. Saline Systems 4:2.

53. Arakawa T, Tokunaga M. 2004. Electrostatic and hydrophobic interactions play a major role in the stability and refolding of halophilic proteins. Protein Pept Lett 11:125-32.

54. Mevarech M, Frolow F, Gloss LM. 2000. Halophilic enzymes: proteins with a grain of salt. Biophys Chem 86:155-64.

55. Eisenberg H. 1995. Life in unusual environments: progress in understanding the structure and function of enzymes from extreme halophilic bacteria. Arch Biochem Biophys 318:1-5.

56. Ortiz-Bermudez P, Srebotnik E, Hammel KE. 2003. Chlorination and cleavage of lignin structures by fungal chloroperoxidases. Appl Environ Microbiol 69:5015-8.

57. Wang G. 2016. Chloride flux in phagocytes. Immunol Rev 273:219-31.

58. Ezraty B, Gennaris A, Barras F, Collet JF. 2017. Oxidative stress, protein damage and repair in bacteria. Nat Rev Microbiol 15:385-396.

59. Stan-Lotter H, Fendrihan S. 2015. Halophilic archaea: life with desiccation, radiation and oligotrophy over geological times. Life (Basel) 5:1487-96.

60. Ghai R, Pašić L, Fernández AB, Martin-Cuadrado AB, Mizuno CM, McMahon KD, Papke RT, Stepanauskas R, Rodriguez-Brito B, Rohwer F, Sánchez-Porro C, Ventosa A, Rodríguez-Valera F. 2011. New abundant microbial groups in aquatic hypersaline environments. Sci Rep 1:135.

61. Kutnowski N, Shmulevich F, Davidov G, Shahar A, Bar-Zvi D, Eichler J, Zarivach R, Shaanan B. 2019. Specificity of protein-DNA interactions in hypersaline environment: 
structural studies on complexes of Halobacterium salinarum oxidative stress-dependent protein hsRosR. Nucleic Acids Res 47:8860-8873.

62. Sharma K, Gillum N, Boyd JL, Schmid A. 2012. The RosR transcription factor is required for gene expression dynamics in response to extreme oxidative stress in a hypersalineadapted archaeon. BMC Genomics 13:351.

63. Tonner PD, Pittman AM, Gulli JG, Sharma K, Schmid AK. 2015. A regulatory hierarchy controls the dynamic transcriptional response to extreme oxidative stress in archaea. PLoS Genet 11:e1004912.

64. Gelsinger DR, Reddy R, Whittington K, Debic S, DiRuggiero J. 2021. Post-transcriptional regulation of redox homeostasis by the small RNA SHOxi in haloarchaea. RNA Biol:115.

65. McMillan LJ, Hwang S, Farah RE, Koh J, Chen S, Maupin-Furlow JA. 2017. Multiplex quantitative SILAC for analysis of archaeal proteomes: a case study of oxidative stress responses. Environ Microbiol.

66. Makarova KS, Wolf YI, Koonin EV. 2015. Archaeal clusters of orthologous genes (arCOGs): An update and application for analysis of shared features between Thermococcales, Methanococcales, and Methanobacteriales. Life (Basel) 5:818-40.

67. Wolf YI, Makarova KS, Yutin N, Koonin EV. 2012. Updated clusters of orthologous genes for Archaea: a complex ancestor of the Archaea and the byways of horizontal gene transfer. Biol Direct 7:46.

68. Szklarczyk D, Gable AL, Lyon D, Junge A, Wyder S, Huerta-Cepas J, Simonovic M, Doncheva NT, Morris JH, Bork P, Jensen LJ, Mering CV. 2019. STRING v11: proteinprotein association networks with increased coverage, supporting functional discovery in genome-wide experimental datasets. Nucleic Acids Res 47:D607-D613. 
69. McMillan LJ, Hwang S, Farah RE, Koh J, Chen S, Maupin-Furlow JA. 2018. Multiplex quantitative SILAC for analysis of archaeal proteomes: a case study of oxidative stress responses. Environ Microbiol 20:385-401.

70. Bailey TL, Johnson J, Grant CE, Noble WS. 2015. The MEME Suite. Nucleic Acids Res 43:W39-49.

71. Grant CE, Bailey TL, Noble WS. 2011. FIMO: scanning for occurrences of a given motif. Bioinformatics 27:1017-8.

72. Kelley LA, Mezulis S, Yates CM, Wass MN, Sternberg MJ. 2015. The Phyre2 web portal for protein modeling, prediction and analysis. Nat Protoc 10:845-58.

73. Baek M, DiMaio F, Anishchenko I, Dauparas J, Ovchinnikov S, Lee GR, Wang J, Cong Q, Kinch LN, Schaeffer RD, Millán C, Park H, Adams C, Glassman CR, DeGiovanni A, Pereira JH, Rodrigues AV, van Dijk AA, Ebrecht AC, Opperman DJ, Sagmeister T, Buhlheller C, Pavkov-Keller T, Rathinaswamy MK, Dalwadi U, Yip CK, Burke JE, Garcia KC, Grishin NV, Adams PD, Read RJ, Baker D. 2021. Accurate prediction of protein structures and interactions using a three-track neural network. Science 373:871-876.

74. Vogt MS, Völpel SL, Albers SV, Essen LO, Banerjee A. 2018. Crystal structure of an Lrs14-like archaeal biofilm regulator from Sulfolobus acidocaldarius. Acta Crystallogr D Struct Biol 74:1105-1114.

75. Zuo G, Chen ZP, Jiang YL, Zhu Z, Ding C, Zhang Z, Chen Y, Zhou CZ, Li Q. 2019. Structural insights into repression of the pneumococcal fatty acid synthesis pathway by repressor FabT and co-repressor acyl-ACP. FEBS Lett 593:2730-2741.

76. Oren A. 2017. Glycerol metabolism in hypersaline environments. Environ Microbiol 19:851-863.

77. Sherwood K, Cano D, Maupin-Furlow J. 2009. Glycerol-mediated repression of glucose metabolism and glycerol kinase as the sole route of glycerol catabolism in the haloarchaeon Haloferax volcanii. J Bacteriol 191:4307-4315. 
78. Saier MH, Ramseier TM. 1996. The catabolite repressor/activator (Cra) protein of enteric bacteria. J Bacteriol 178:3411-7.

79. Martinez-Pastor M, Tonner PD, Darnell CL, Schmid AK. 2017. Transcriptional regulation in archaea: From individual genes to global regulatory networks. Annu Rev Genet 51:143-170.

80. Toledano MB, Kullik I, Trinh F, Baird PT, Schneider TD, Storz G. 1994. Redoxdependent shift of OxyR-DNA contacts along an extended DNA-binding site: a mechanism for differential promoter selection. Cell 78:897-909.

81. Li Y, He ZG. 2012. The mycobacterial LysR-type regulator OxyS responds to oxidative stress and negatively regulates expression of the catalase-peroxidase gene. PLoS One 7:e30186.

82. D'Autréaux B, Toledano MB. 2007. ROS as signalling molecules: mechanisms that generate specificity in ROS homeostasis. Nat Rev Mol Cell Biol 8:813-24.

83. Okazaki S, Tachibana T, Naganuma A, Mano N, Kuge S. 2007. Multistep disulfide bond formation in Yap1 is required for sensing and transduction of $\mathrm{H}_{2} \mathrm{O}_{2}$ stress signal. Mol Cell $27: 675-88$.

84. Delaunay A, Isnard AD, Toledano MB. 2000. $\mathrm{H}_{2} \mathrm{O}_{2}$ sensing through oxidation of the Yap1 transcription factor. EMBO J 19:5157-66.

85. Paulsen CE, Carroll KS. 2009. Chemical dissection of an essential redox switch in yeast. Chem Biol 16:217-25.

86. Napoli A, van der Oost J, Sensen CW, Charlebois RL, Rossi M, Ciaramella M. 1999. An Lrp-like protein of the hyperthermophilic archaeon Sulfolobus solfataricus which binds to its own promoter. J Bacteriol 181:1474-80.

87. Bell SD, Jackson SP. 2000. Mechanism of autoregulation by an archaeal transcriptional repressor. J Biol Chem 275:31624-9. 
88. Fiorentino G, Cannio R, Rossi M, Bartolucci S. 2003. Transcriptional regulation of the gene encoding an alcohol dehydrogenase in the archaeon Sulfolobus solfataricus involves multiple factors and control elements. J Bacteriol 185:3926-34.

89. Napoli A, Kvaratskelia M, White MF, Rossi M, Ciaramella M. 2001. A novel member of the bacterial-archaeal regulator family is a nonspecific dna-binding protein and induces positive supercoiling. J Biol Chem 276:10745-52.

90. Orell A, Peeters E, Vassen V, Jachlewski S, Schalles S, Siebers B, Albers SV. 2013. Lrs14 transcriptional regulators influence biofilm formation and cell motility of Crenarchaea. ISME J 7:1886-98.

91. Koerdt A, Orell A, Pham TK, Mukherjee J, Wlodkowski A, Karunakaran E, Biggs CA, Wright PC, Albers SV. 2011. Macromolecular fingerprinting of Sulfolobus species in biofilm: a transcriptomic and proteomic approach combined with spectroscopic analysis. J Proteome Res 10:4105-19.

92. Li L, Banerjee A, Bischof LF, Maklad HR, Hoffmann L, Henche AL, Veliz F, Bild W, Schulte U, Orell A, Essen LO, Peeters E, Albers SV. 2017. Wing phosphorylation is a major functional determinant of the Lrs14-type biofilm and motility regulator AbfR1 in Sulfolobus acidocaldarius. Mol Microbiol 105:777-793.

93. Sakrikar S, Schmid AK. 2021. An archaeal histone-like protein regulates gene expression in response to salt stress. Nucleic Acids Res 49:12732-12743.

94. Martin JH, Sherwood Rawls K, Chan JC, Hwang S, Martinez-Pastor M, McMillan LJ, Prunetti L, Schmid AK, Maupin-Furlow JA. 2018. GlpR Is a direct transcriptional repressor of fructose metabolic genes in Haloferax volcanii. J Bacteriol 200:e00244-18.

95. Schulze S, Adams Z, Cerletti M, De Castro R, Ferreira-Cerca S, Fufezan C, Giménez MI, Hippler M, Jevtic Z, Knüppel R, Legerme G, Lenz C, Marchfelder A, Maupin-Furlow J, Paggi RA, Pfeiffer F, Poetsch A, Urlaub H, Pohlschroder M. 2020. The Archaeal 
Proteome Project advances knowledge about archaeal cell biology through comprehensive proteomics. Nat Commun 11:3145.

96. Deatherage DE, Barrick JE. 2014. Identification of mutations in laboratory-evolved microbes from next-generation sequencing data using breseq. Methods Mol Biol $1151: 165-88$.

97. Wilbanks EG, Larsen DJ, Neches RY, Yao Al, Wu CY, Kjolby RA, Facciotti MT. 2012. A workflow for genome-wide mapping of archaeal transcription factors with ChIP-seq. Nucleic Acids Res 40:e74.

98. Langmead B, Salzberg SL. 2012. Fast gapped-read alignment with Bowtie 2. Nat Methods 9:357-9.

99. Li H, Handsaker B, Wysoker A, Fennell T, Ruan J, Homer N, Marth G, Abecasis G, Durbin R, Subgroup GPDP. 2009. The Sequence Alignment/Map format and SAMtools. Bioinformatics 25:2078-9.

100. Kuan PF, Chung D, Pan G, Thomson JA, Stewart R, Keleş S. 2011. A statistical framework for the analysis of ChIP-seq data. J Am Stat Assoc 106:891-903.

101. Carroll TS, Liang Z, Salama R, Stark R, de Santiago I. 2014. Impact of artifact removal on ChIP quality metrics in ChIP-seq and ChIP-exo data. Front Genet 5:75.

102. Ross-Innes CS, Stark R, Teschendorff AE, Holmes KA, Ali HR, Dunning MJ, Brown GD, Gojis O, Ellis IO, Green AR, Ali S, Chin SF, Palmieri C, Caldas C, Carroll JS. 2012. Differential oestrogen receptor binding is associated with clinical outcome in breast cancer. Nature 481:389-93.

103. Yu G, Wang LG, He QY. 2015. ChIPseeker: an R/Bioconductor package for ChIP peak annotation, comparison and visualization. Bioinformatics 31:2382-3.

104. Robinson JT, Thorvaldsdóttir H, Winckler W, Guttman M, Lander ES, Getz G, Mesirov JP. 2011. Integrative genomics viewer. Nat Biotechnol 29:24-6. 
105. Darnell CL, Tonner PD, Gulli JG, Schmidler SC, Schmid AK. 2017. Systematic discovery of archaeal transcription factor functions in regulatory networks through quantitative phenotyping analysis. mSystems 2.

106. Darnell CL, Schmid AK. 2015. Systems biology approaches to defining transcription regulatory networks in halophilic archaea. Methods 86:102-14.

107. Altschul SF, Gish W, Miller W, Myers EW, Lipman DJ. 1990. Basic local alignment search tool. J Mol Biol 215:403-10.

108. Pettersen EF, Goddard TD, Huang CC, Meng EC, Couch GS, Croll TI, Morris JH, Ferrin TE. 2021. UCSF ChimeraX: Structure visualization for researchers, educators, and developers. Protein Sci 30:70-82.

109. Edgar R, Domrachev M, Lash AE. 2002. Gene Expression Omnibus: NCBI gene expression and hybridization array data repository. Nucleic Acids Res 30:207-10.

110. Barrett T, Wilhite SE, Ledoux P, Evangelista C, Kim IF, Tomashevsky M, Marshall KA, Phillippy KH, Sherman PM, Holko M, Yefanov A, Lee H, Zhang N, Robertson CL, Serova N, Davis S, Soboleva A. 2013. NCBI GEO: archive for functional genomics data sets-update. Nucleic Acids Res 41:D991-5.

111. Leinonen R, Sugawara H, Shumway M, Collaboration INSD. 2011. The sequence read archive. Nucleic Acids Res 39:D19-21. 
Table 1. Gene loci with 5' regions detected as high peaks by OxsR ChIP seq analysis'.

\begin{tabular}{|c|c|c|c|c|}
\hline Gene locus $F$ & Protein names & $\mathrm{NaOCl}(-)$ & $\mathrm{NaOCl}(+)$ & High peak \\
\hline HVO_1043* & DUF1684 family protein & $\mathrm{V}$ & $\mathrm{V}$ & V \\
\hline HVO_A0618*C & Coenzyme A disulfidereductase homolog & $\mathrm{V}$ & $\mathrm{V}$ & $\mathrm{V}$ \\
\hline HVO_1875 & $\begin{array}{l}\text { Galactoside O-acetyltransferase (EC 2.3.1.-) } \\
\text { homolog }\end{array}$ & $\mathrm{V}$ & $\mathrm{V}$ & V \\
\hline HVO_0198 L & UPF0213 family protein & $\mathrm{V}$ & $\mathrm{V}$ & $\mathrm{V}$ \\
\hline HVO_1342* & Thioredoxin & $\mathrm{V}$ & $\mathrm{V}$ & $\mathrm{V}$ \\
\hline HVO_0811* L & L-Aspartate decarboxylase (EC 4.1.1.11) homolog & & $\mathrm{V}$ & V \\
\hline HVO_0337 C & Glutaredoxin & & $\mathrm{V}$ & V \\
\hline HVO_0040* 1 & Transmembrane protein & & $\mathrm{V}$ & V \\
\hline HVO_0004 & NamA family oxidoreductase & & $\mathrm{V}$ & V \\
\hline HVO_1031 T & Thioredoxin-disulfide reductase (EC 1.8.1.9) & & $\mathrm{V}$ & V \\
\hline HVO_1166 & $\begin{array}{l}\text { Chloroplast RNA splicing and ribosome maturation } \\
\text { (CRM) domain protein }\end{array}$ & & $\mathrm{V}$ & V \\
\hline HVO_1684 T & Threonine-tRNA ligase (EC 6.1.1.3) & & $\mathrm{V}$ & V \\
\hline HVO_0497 C & Cold shock protein & & $\mathrm{V}$ & V \\
\hline HVO_0251 C & Glutaredoxin & & $\mathrm{V}$ & V \\
\hline HVO_1244 T & Thioredoxin & & $\mathrm{V}$ & V \\
\hline HVO_1668* Y & v-Glutamylcysteine synthetase (EC 6.3.2.2) & & $\mathrm{V}$ & V \\
\hline HVO_1522 ト & Hexuronic acid methyltransferase AgIP (EC 2.1.1.-) & & $\mathrm{V}$ & V \\
\hline HVO_1438* Y & YneT family protein & & $\mathrm{V}$ & $\mathrm{V}$ \\
\hline HVO_1457 C & Glycoside hydrolase domain protein & & V & V \\
\hline HVO_2157 L & Uncharacterized protein & & V & V \\
\hline HVO_2859 [ & DUF63 family protein & & V & V \\
\hline HVO_1124 [ & DUF357 family protein & & & V \\
\hline HVO_1017 r & Ketosamine kinase domain protein & & & V \\
\hline
\end{tabular}

${ }^{1}$ Blue highlight, putative function in low molecular weight thiol (LMWT) biosynthesis or thiol relay; *, CGrich DNA motif $\underline{\mathrm{CG}} \underline{\mathrm{CG}} \underline{\mathrm{CCG}}$ identified 5' of these gene homologs. V, present in the condition. 


\section{Figures and legends}

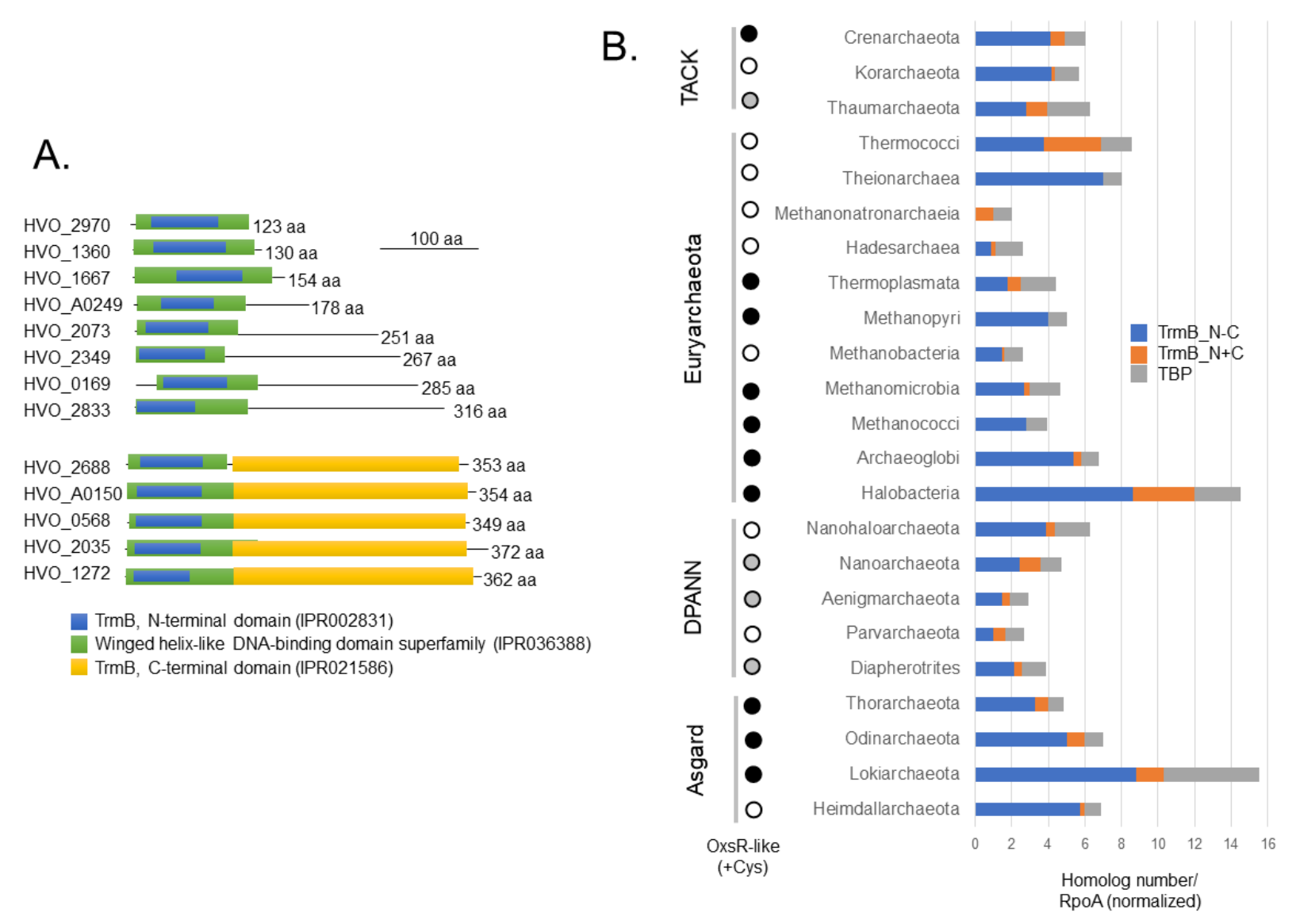

Figure 1. Haloferax volcanii OxsR (HVO_2970) is a member of the TrmB family and is conserved across multiple phyla of Archaea. A) Two major types of TrmB family members observed. $H$. volcanii TrmB family proteins with: i) an N-terminal winged helix-turn-helix (wHTH) DNA binding domain (green and blue) and a C-terminal ligand (sugar) binding domain (yellow) and ii) only the wHTH domain. OxsR is of the latter type. B) Distribution of OxsR and TrmB family homologs among archaea. TrmB family proteins with only an N-terminal DNA binding domain (IPR002831) (blue bar), TrmB proteins with an N-terminal DNA binding domain and C-terminal ligand sensing domain (IPR021586) (orange bar), and TATA binding proteins (TBPs, IPR000814) (grey bar) are compared as ratios of UniProt hits per RNA polymerase alpha subunit (RpoA, IPR000722) to account for differences in genome sequence availability among the phyla. Among the archaea, TrmB homologs with a C-terminal ligand sensing domain were not detected in Methanopyri, Methanococci and Theionarchaea. Archaea with cysteine-containing OxsR homologs are indicated by black and grey circles (with grey indicating that many archaea within this group do not contain OxsR homologs). 

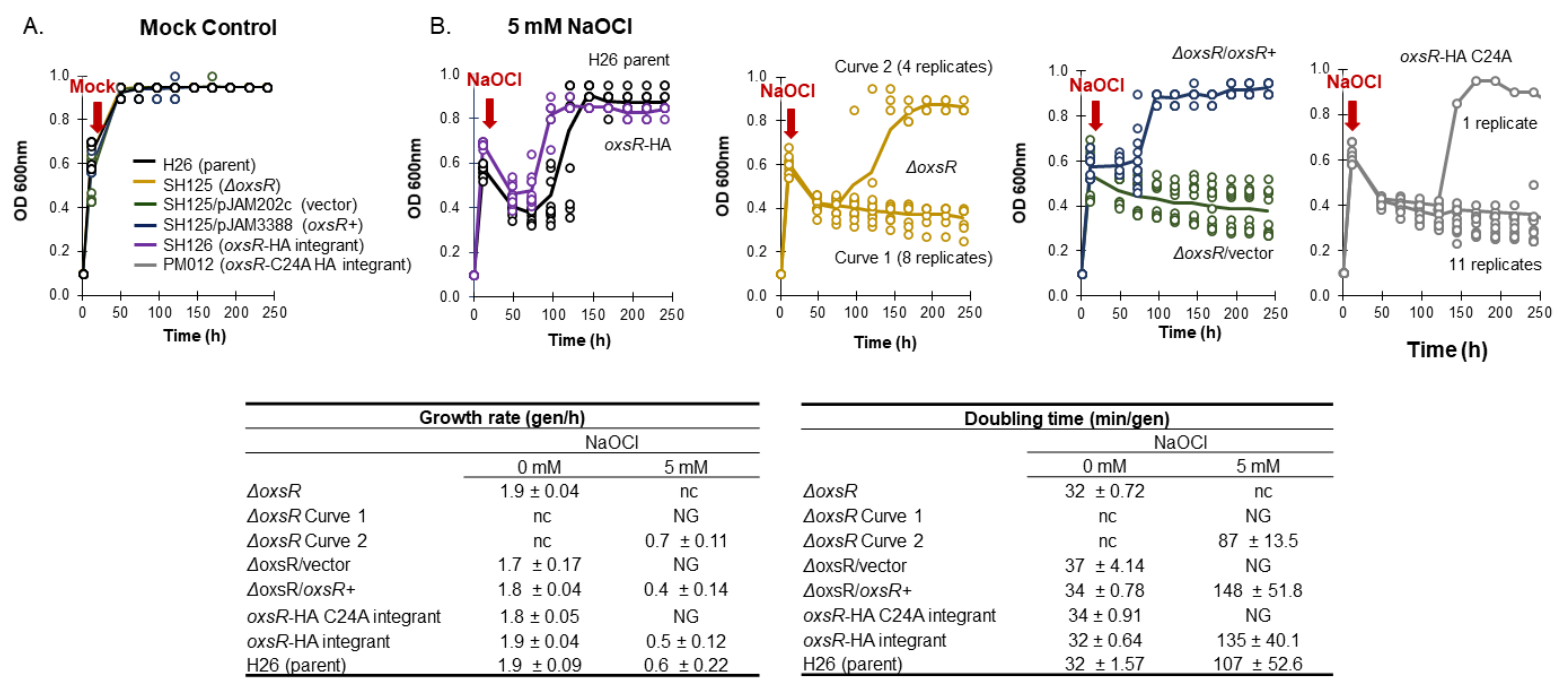

$\mathrm{nc}=$ not calculated

$\mathrm{NG}=$ no growth

Figure 2. OxsR facilitates recovery of $H$. volcanii from hypochlorite stress. Strains were grown glycerol minimal medium (GMM) to log-phase $(11 \mathrm{hr}, \mathrm{OD} 600 \mathrm{~nm}, 0.5-0.6)$ and then treated with a mock control (A) or $5 \mathrm{mM} \mathrm{NaOCl}$ (B) as indicated. Lower panels, Growth rates (left) and doubling times (right) are given for each strain under optimal conditions $(0 \mathrm{mM})$ or hypochlorite stress $(5 \mathrm{mM} \mathrm{NaOCl})$. The markers represent the individual replicates ( $n=12$ total; 6 replicates $x 2$ experiments). The colored curves represent the average growth for the 12 replicates with exception of the $\mathrm{NaOCl}$ treated $\Delta$ oxs $R$ mutant and oxs $R$-HA C24A integrant strains which are represented by two curves. Cell growth was monitored at OD600 nm by direct measurement in the $13 \times 100 \mathrm{~mm}$ culture tubes using a Spectronic 20+ spectrophotometer (ThermoSpectronic, Filter: $600-950 \mathrm{~nm}$ ). The measurement plateau at OD600 of 0.95 is the maximum OD600 the spectrophotometer could read. See methods for details. 


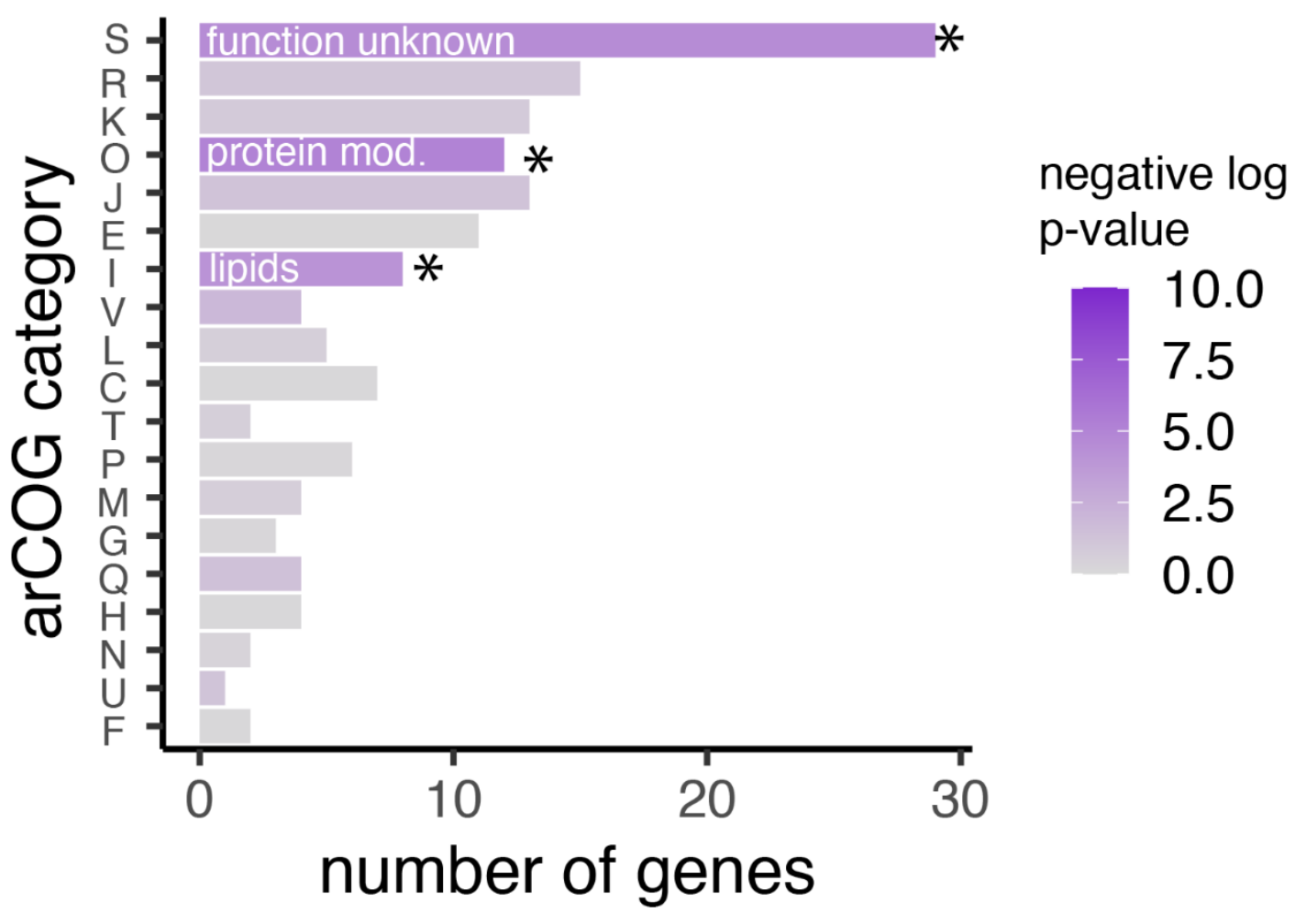

Figure 3. General functional classification of genes predicted to be bound by OxsR according to ChIPseq data. Gene homologs flanking the intergenic regions of DNA detected to be bound by OxsR through ChIP-seq analysis were clustered by arCOG according to function. Bar lengths represent the number of genes detected in each functional category, and the shading of the bar represents the significance of enrichment in each category (see legend). 

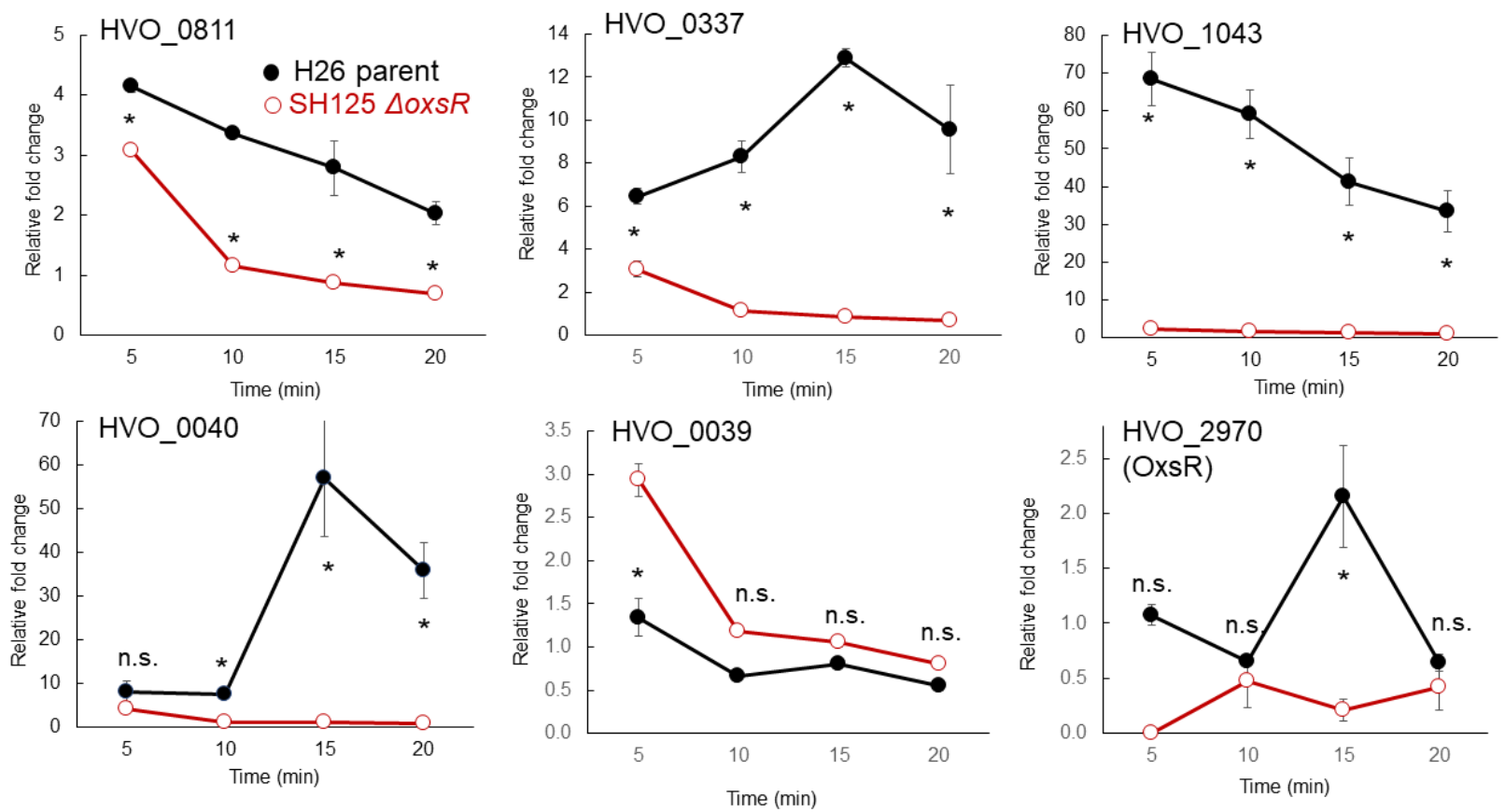

Figure 4. Influence of hypochlorite stress and oxs $R$ on the expression of select genes identified by ChIPseq analysis. Relative-fold change represents the transcript abundance ratio of $\mathrm{NaOCl}$ : mock treated cells. The $H$. volcanii $\mathrm{H} 26$ parent and $\mathrm{SH} 125(\Delta o x s R)$ mutant cells were grown in GMM to exponential phase and treated with 0 and $2.5 \mathrm{mM} \mathrm{NaOCl}$ for 5, 10, 15 and 20 min. Total RNA was extracted and used for quantitative real-time (qRT) PCR. Levels of the gene expression were normalized to the internal reference ribL (HVO_1015, one-fold). Targets of qRT-PCR are indicated as gene locus tag numbers within each panel. *Significant differences between the parent and mutant by the student t-test analysis (p-value $\leq 0.05$ ). n.s., not significant. All data are expressed as mean \pm S.E.M. See methods for details. 
A.

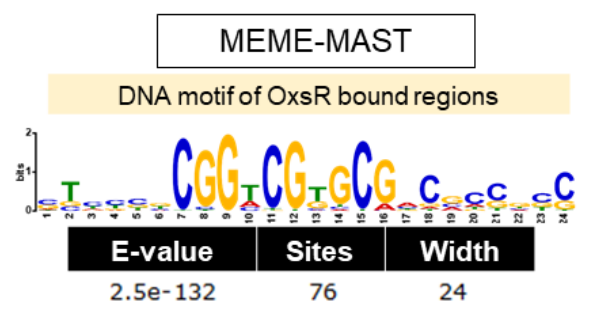

B.
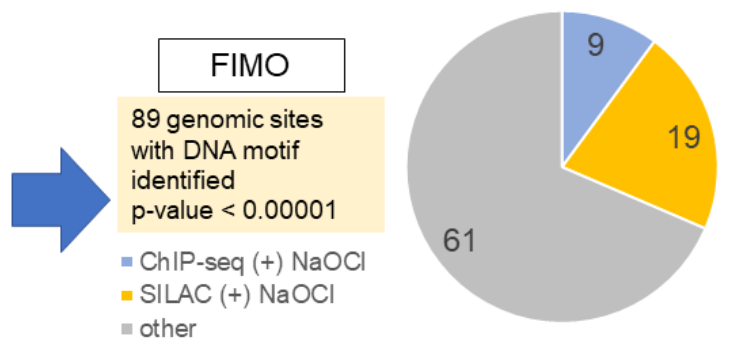

\begin{tabular}{|c|c|c|c|c|c|}
\hline Gene locus & Description of gene & Strand & p-value & q-value & Matched Sequence \\
\hline \multicolumn{6}{|c|}{ Top FIMO hits: } \\
\hline HVO_1342 & Thioredoxin & + & $4.43 \mathrm{E}-10$ & 0.00116 & GTTTCGCGGTCGTGCGTCGCCGCG \\
\hline HVO_1043 & DUF1684 family protein & + & 4.33E-08 & 0.0397 & GTCCGCCGGTCGTGCGTCCCCCGC \\
\hline HVO_0039 & DNA repair helicase Rad3b (EC 3.6.4.12) & + & $6.06 \mathrm{E}-08$ & 0.0397 & GTCACTCGGACGGGCGGCACCGCC \\
\hline HVO_0040 & Uncharacterized TM protein & - & $6.06 \mathrm{E}-08$ & 0.0397 & GTCACTCGGACGGGCGGCACCGCC \\
\hline HVO_1442 & ABC-type permease & - & $9 \mathrm{E}-08$ & 0.0472 & GTCCGGCGGTCGAGCGTCCCCGCC \\
\hline HVO_1668 & $\begin{array}{l}\text { Y-glutamylcysteine synthetase (EC } 6.3 .2 .2 \text { ) } \\
\text { arCOG06248 protein (intergenic region shared with }\end{array}$ & + & $1.14 \mathrm{E}-07$ & 0.0494 & CGGCGGCGGTCGTGCGACGCGTTC \\
\hline HVO 2897 & HVO 2896 A/G-specific adenine glycosylase) & - & 1.32E-07 & 0.0494 & GTCTCGCGGTCGAGCGACACGTCG \\
\hline
\end{tabular}

C.

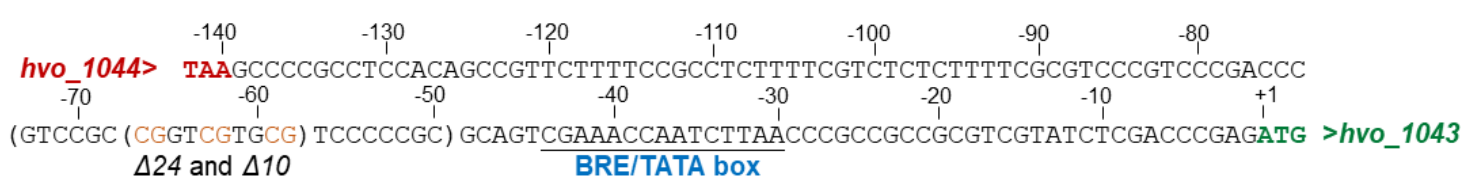

D.
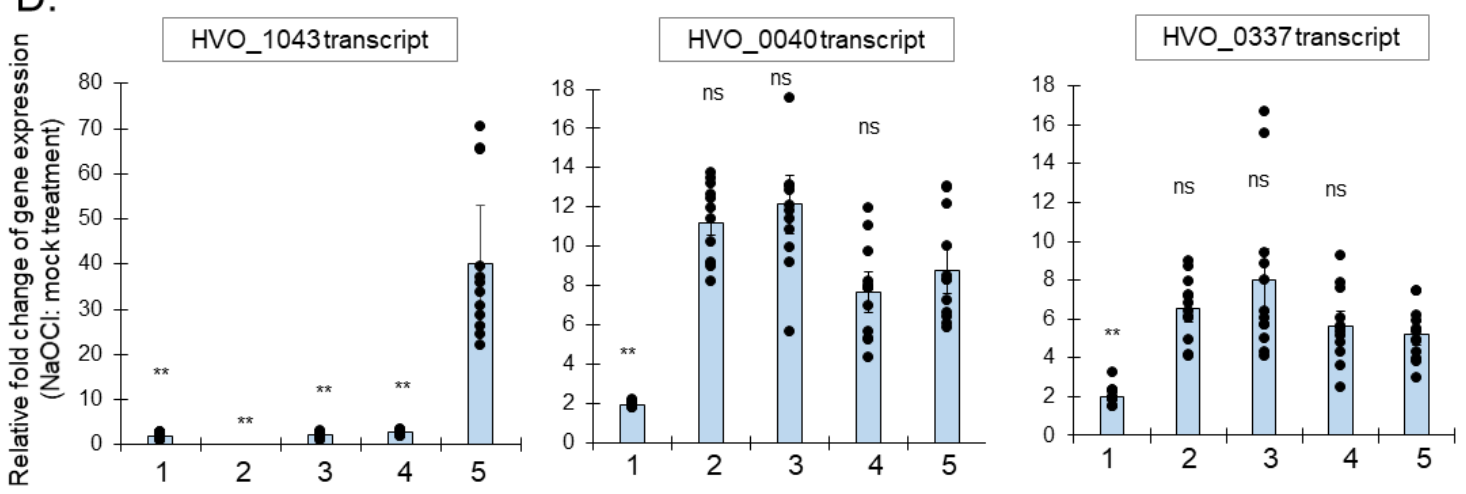

1. $\Delta \mathrm{OxSR}(\mathrm{SH} 125)$

2. $\Delta$ hvo_1043(PM57)

3. $\Delta 24$ bp CG 5' hvo_1043 (PM58)

4. $\triangle 10$ bp CG 5' hvo_1043 (PM59)

Figure 5. CG-rich DNA motif associated with OxsR-mediated transcriptional activation. A) CG-rich motif identified to be enriched in OxsR-bound ChIP-seq DNA sequences. The motif was identified by MEMEMAST analysis and was input into FIMO to scan the $H$. volcanii genome. The 89 sites identified by this approach at a $p$-value $<0.00001$ were compared to the OxsR ChIP-seq (blue) and SILAC-based MS (orange) datasets. B) FIMO hits of the CG-rich motif detected at a $q$-value $<0.05$ with the sites common to the ChIP-seq (blue) and SILAC-based MS (orange) datasets highlighted. C) CG-rich DNA motif upstream of the BRE/TATA box of $h v o \_1043$ targeted for deletion. Parenthesis, CG-rich repeat region targeted for mutagenesis with the CG repeat in orange ( $\Delta 24$ spans -50 to -74 and $\Delta 10$ spans -58 to -67 ). Red, stop codon of $h v o \_1044$. Underlined, BRE/TATA box region. Green, start codon of $h v o \_1043$. D. qRT-PCR analysis of deletions of CG-rich repeat 5' of hvo_1043. $\mathrm{H}$. volcanii strains were grown in GMM to early log phase (OD600 of 0.3 to 0.5 ) and exposed to $2.5 \mathrm{mM} \mathrm{NaOCl}$ or a mock control for $15 \mathrm{~min}$. Transcript levels were monitored in the mutant strains as indicated. The internal reference hvo_1015 normalized levels of gene expression were at one-fold relative fold change. Targets of qRT-PCR are indicated for each panel by gene locus tag number. Strains used for preparation of RNA are indicated on the $x$-axis. Significant differences between the parent and mutants by the student t-test analysis $\left({ }^{* *}, p\right.$ value $\leq 0.01$; n.s., not significant) (Exp./Bio: 4 ; Tech: 3 replicates). See methods for details. 

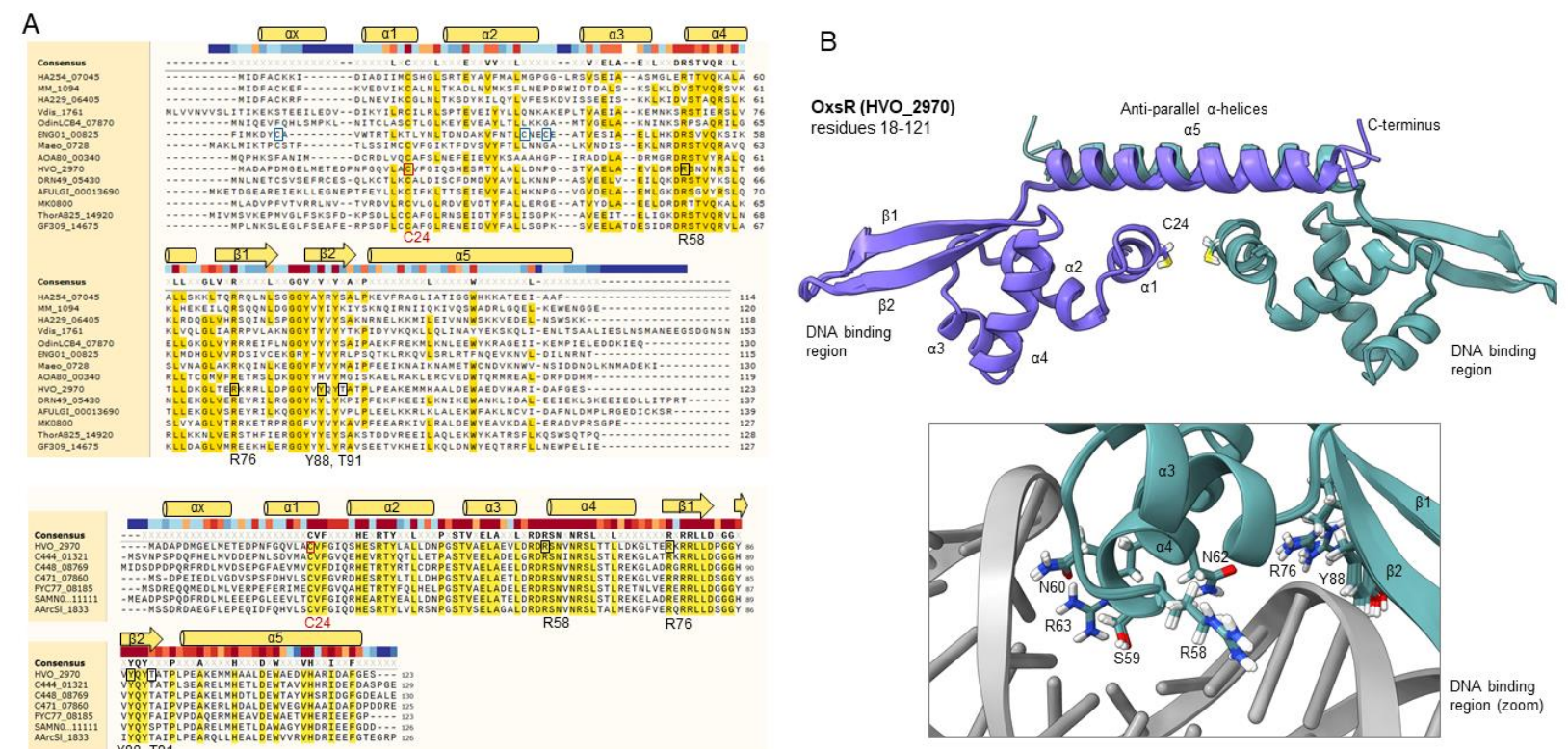

Figure 6. Conserved residues and 3D-structural model of OxsR. A) Multiple amino acid sequence alignment of OxsR (HVO_2970) to TrmB family proteins. Upper: OxsR aligned to representative homologs from diverse archaea. Crenarchaeota (Vdis_1761), Thaumarchaeota (DRN49_05430), Thermoplasmata (AOA80_00340), Methanopyri (MK0800), Methanomicrobia (MM_1094), Methanococci (Maeo_0728), Archaeoglobi (AFULGI_00013690), Nanoarchaeota (HA229_06405), Aenigmarchaeota (ENG01_00825), Diapherotrites (HA254_07045), Thorarchaeota (ThorAB25_14920), Odinarchaeota (OdinLCB4_07870) and Lokiarchaeota (GF309_14675). Lower: OxsR aligne-d to representative homologs from diverse families of haloarchaea. Halorubraceae (C471_07860 and AArcSI_1833), Natrialbaceae (FYC77_08185), Haloarculaceae (C444_01321), Halococcaceae (C448_08769), and Halobacteriaceae (SAMNN05216226_111111). Residues of OxSR discussed in text indicated by black and red boxes and numbered below the alignment. Blue boxes indicate cysteine residues in the $\mathrm{N}$-terminal region of Aenigmarchaeota homolog (ENG01_00825). Predicted $\alpha$ helices and $\beta$ strands of OxsR indicated above the alignment. Residues at $>50 \%$ and $>95 \%$ amino acid sequence identity are indicated by yellow highlighting (upper and lower panels, respectively) with the bar colored dark red to dark blue above the sequence indicating residues of high to low conservation. B) 3D-structural model of OxsR (HVO_2970). Ribbon diagram of OxsR in homodimeric configuration (chain A and B in purple and cadet blue, respectively). Residue numbering and secondary structure indicated for chain A. The 3D-structural model generated by RoseTTAfold for residues 18-123 is displayed. Arrangement of the 3D-model into a homodimer was by comparison to the X-ray crystal structure of the biofilm regulator Sulfolobus acidocaldarius AbfR2 (Saci_1223; PDB: 6CMV). DNA interactions were predicted by comparison to the Xray crystal structure of the Streptococcus pneumoniae FabT:DNA complex (PDB: 6JBX). 
A

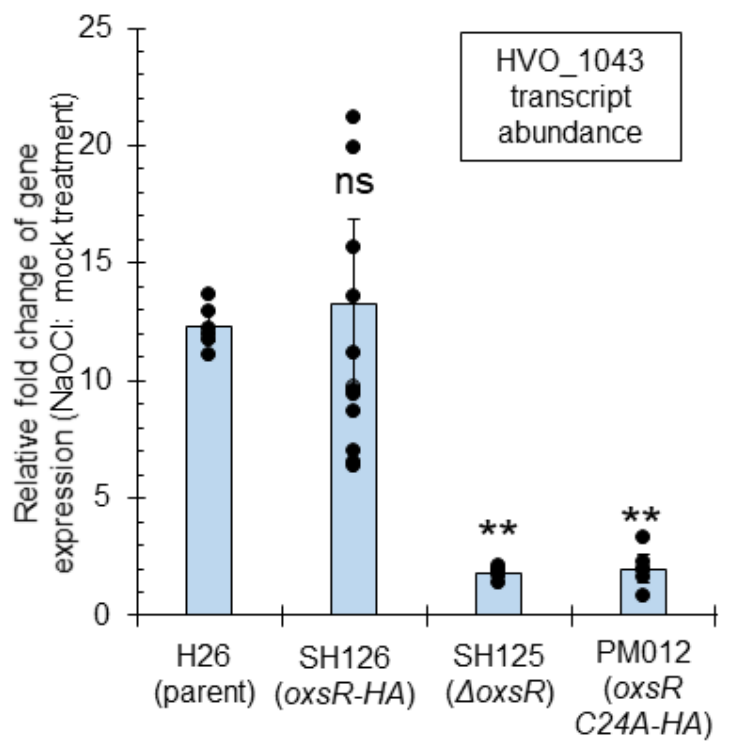

B

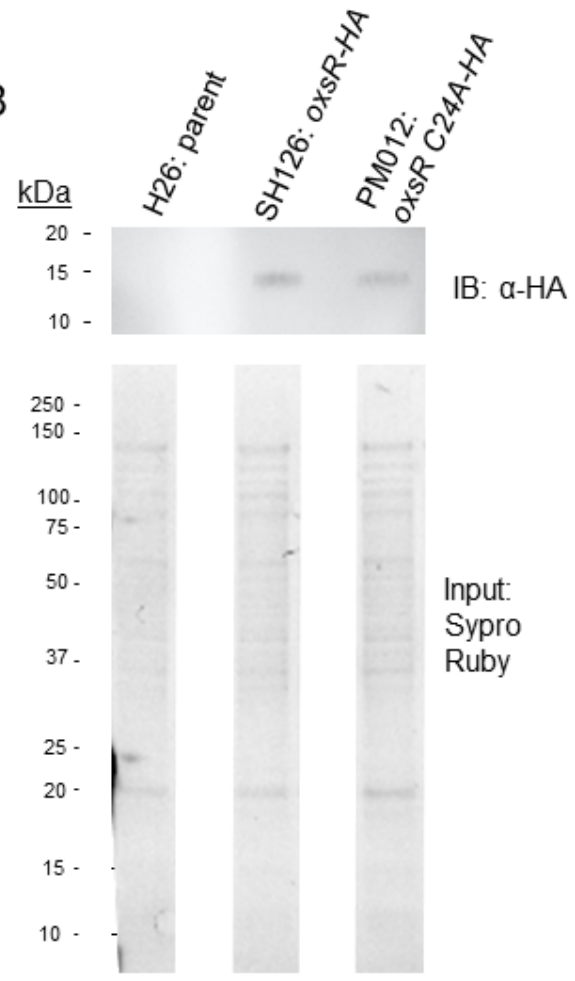

Figure 7. Conserved cysteine residue (C24) important for OxsR function. A) Relative-fold change of hvo_1043 transcript levels during hypochlorite stress. H. volcanii strains as indicated on the $x$-axis were grown to early exponential phase in GMM and treated with 0 and $2.5 \mathrm{mM} \mathrm{NaOCl}$ for $15 \mathrm{~min}$. This timeframe was found to result in a 10- to 40-fold increased abundance of hvo_1043 transcripts in the parent strain. Total RNA was extracted and used for qRT-PCR analysis. The internal reference hvo_1015 normalized levels of the gene expression were at one-fold relative fold change. Significant differences between the parent and mutant by the student t-test analysis ( ${ }^{* *}, p$-value $\leq 0.001 ;{ }^{*}, p$-value $\leq 0.05$ ). n.s., not significant. (Exp./Bio: 2-4; Tech: 3 replicates). B) Detection of OxsR-HA with and without the C24A variant in $H$. volcanii cells. Strains were grown to early log phase (OD600 of 0.3 to 0.5$)$. Upper panel: Immunoprecipitates ( $10 \mu \mathrm{l}$ per lane) were separated by reducing 15\% SDS-PAGE for $2 \mathrm{~h}$ at $100 \mathrm{~V}$. Proteins were detected by immunoblotting analysis using anti-HA tag HRP (\# ab1190) antibodies at 1:20,000 dilution and ECL Prime. Signal was visualized after a $30 \mathrm{sec}$ exposure. Molecular mass standards were Precision Plus Protein Kaleidoscope. Lower panel: Total protein input separated by reducing $12 \%$ SDS-PAGE prior to immunoprecipitation detected by Sypro Ruby staining is included as control. Samples were normalized as $4 \mu \mathrm{l}\left(0.04 \mathrm{OD}_{600}\right.$ units cell pellet) per lane. See methods for details. 


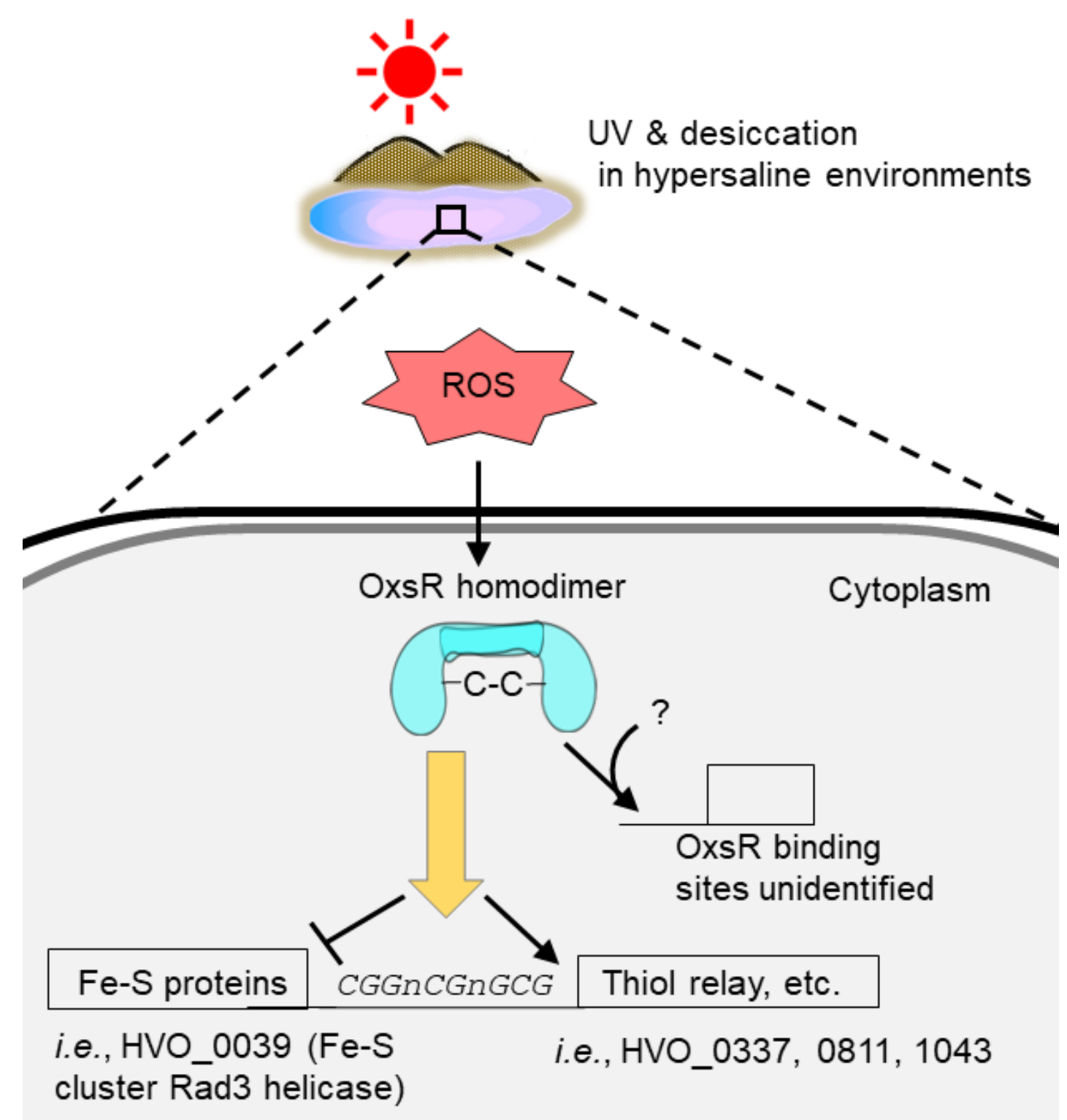

Figure 8. A proposed model of OxsR-mediated oxidative response in $H$. volcanii. The diagram shows that ROS generated from (a)biotic reactions is sensed by OxsR and then an intersubunit disulfide bond is formed at the conserved cysteine residue, followed by regulating gene expression (activation/repression and target DNA binding). 